\title{
DEGENERATE EVOLUTION EQUATIONS IN HILBERT SPACE
}

\author{
BY \\ AVNER FRIEDMAN $\left({ }^{1}\right)$ AND ZEEV SCHUSS
}

\begin{abstract}
We consider the degenerate evolution equation $c_{1}(t) d u / d t+c_{2}(t) A(t) u$ $=f(t)$ in Hilbert space, where $c_{1} \geqq 0, c_{2} \geqq 0, c_{1}+c_{2}>0 ; A(t)$ is an unbounded linear operator satisfying the usual conditions which ensure that there is a unique solution for the Cauchy problem $d u / d t+A(t) u=f(t)$ in $(0, T], u(0)=u_{0}$. We prove the existence and uniqueness of a weak solution, and differentiability theorems. Applications to degenerate parabolic equations are given.
\end{abstract}

Introduction. Consider an evolution equation

$$
c_{1}(t) \frac{d u}{d t}+c_{2}(t) A(t) u=f(t) \quad \text { in }(0, T]
$$

in Hilbert space $X$, where $A(t)$ is an unbounded linear operator satisfying the standard assumptions which ensure that the Cauchy problem

$$
\frac{d u}{d t}+A(t) u=f(t) \quad \text { in }(0, T], \quad u(0)=u_{0},
$$

has a unique solution. If, for each $t \in[0, T], c_{1}(t) \geqq 0, c_{2}(t) \geqq 0, c_{1}(t)+c_{2}(t)>0$, then we call $(0.1)$ a degenerate evolution equation.

The aim of this paper is to prove existence, uniqueness and differentiability theorems for solutions of the degenerate evolution equation (0.1) (under suitable initial conditions).

In $\$ \S 1-5$ we consider the special case where $c_{2} \equiv 1$. In $\S 1$ we define the concept of a weak solution and prove that it is a classical solution in every interval where $c_{1}>0$. It is also shown that a weak solution in $[0, T]$ is a weak solution in any subinterval $[a, b]$ of $[0, T]$. In $\S 2$ we prove that a weak solution exists. In $\S 3$ it is proved that the weak solution is unique. Finally, in $\$ 4$ we prove differentiability theorems for the weak solution. In $\S 5$ we give another proof of uniqueness, based

Received by the editors July 22, 1970.

AMS 1969 subject classifications. Primary 3563, 3565, 3495; Secondary 3524, 3531, 3537, $4690,4765$.

Key words and phrases. Evolution equations, degenerate equation, Cauchy problem, weak solution, existence, uniqueness, differentiability of solutions, parabolic equations.

( ${ }^{1}$ ) The first author is partially supported by the National Science Foundation Grant NSF GP-5558.

Copyright (C) 1971, American Mathematical Society 
on an a priori inequality for weak solutions. Unlike the first proof, the second proof extends to degenerate parabolic equations, as well as to equations $(0.1)$ with $c_{1} \geqq 0$, $c_{2} \geqq 0, c_{1}+c_{2}>0$.

Degenerate parabolic equations with $c_{1}=c_{1}(x, t) \geqq 0$ and $c_{2}=c_{2}(x, t) \equiv 1$ are treated in $\S 6$.

In $\S 7$ we consider $(0.1)$ with $c_{1} \equiv 1$ and $c_{2} \geqq 0$. We prove existence, uniqueness and differentiability theorems. The analogous parabolic case is also treated.

The general case of $(0.1)$ is considered in $\S 8$.

The results of this paper, when $A(t)$ is specialized to a second order elliptic operator, overlap with known theorems for second order degenerate ellipticparabolic equations. Such equations were first treated by Fichera [1], [2], who proved the existence of weak solutions and derived $L^{p}$ estimates. Regularity and uniqueness theorems have since been derived by several authors, in particular, by Oleǐnik [7] and, most extensively, by Kohn-Nirenberg [6].

We also mention the paper of Gluško-Krein [5] announcing results concerning the evolution equation (0.1) with $c_{2} \equiv 1, c_{1}(0)=0, c_{1}(t)>0$ if $t>0$; the operator $A(t)$, in [5], is a bounded operator.

1. Weak solutions: general properties. Let $X$ be a complex Hilbert space with inner product $($,$) and norm |\cdot|$.

Definition 1.1. An operator valued function $A(t)$ on $[0, T]$ is said to belong to the class $\mathfrak{A}_{0}$ if it satisfies the following conditions:

(i) The domain $D_{A}$ of $A(t)(0 \leqq t \leqq T)$ is dense in $X$ and is independent of $t$, and $A(t)$ is a closed linear operator from $D_{A}$ into $X$.

(ii) For each $t \in[0, T]$ the resolvent $R(\lambda, A(t))$ of $A(t)$ exists for all $\lambda$ with $\operatorname{Re} \lambda \leqq 0$, and $\|R(\lambda, A(t))\| \leqq C /(1+|\lambda|)(\operatorname{Re} \lambda \leqq 0)$ where $C$ is some positive constant;

(iii) For any $t, s, \tau$ in $[0, T]$,

$$
\begin{aligned}
\left\|[A(t)-A(\tau)] A^{-1}(s)\right\| & \leqq C|t-\tau|^{\alpha}, \\
\left\|A^{-1}(s)[A(t)-A(\tau)] x\right\| & \leqq C|t-\tau|^{\alpha}\|x\| \quad\left(x \in D_{A}\right) ;
\end{aligned}
$$

the constants $C, \alpha$ are independent of $t, s, \tau$, and $0<\alpha \leqq 1$.

Definition 1.2. Let $c(t)$ be a real valued function on $[0, T]$ that satisfies the conditions

(i) $c(t) \geqq 0$ for all $t \in[0, T]$;

(ii) $c(t)$ is continuous and $c^{\prime}(t)$ is piecewise continuous in $[0, T]$.

Such a function is said to belong to the class $\mathscr{C}_{0}$.

Definition 1.3. Let $A(t) \in \mathfrak{A}_{0}$ and satisfy the condition $\operatorname{Re}(A(t) u, u) \geqq \gamma|u|^{2}$ for all $u \in D_{A}$, where $\gamma>0, \gamma \geqq \frac{1}{2} c^{\prime}(t)+\eta, \eta>0$ for all $t \in[0, T]$. Then $A(t)$ is said to satisfy the condition $\left(\mathrm{AC}_{0}\right)$.

Definition 1.4. Let $f(t) \in C([0, T], X)$. An $X$-valued function $u(t)$ is said to be a classical solution of

$$
c(t) u^{\prime}(t)+A(t) u(t)=f(t) \quad \text { in }(a, b) \subset(0, T)
$$


if $u(t) \in C^{1}((a, b), X), u(t) \in D_{A}$ for all $t \in(a, b), A(t) u(t) \in C((a, b), X)$ and $u(t)$ satisfies $(1.1)$ in $(a, b)$.

Definition 1.5. Let $\varphi(t) \in C^{1}([a, b], X), \varphi(t) \in D_{A^{*}(t)}$ for all $t \in[a, b], \varphi(b)=0$, and $A^{*}(t) \varphi(t) \in C([a, b], X)$; here $A^{*}(t)$ is the adjoint of $A(t)$ and $D_{A^{*}(t)}$ is its domain. We then say that $\varphi$ is a test function in $[a, b]$.

Definition 1.6. Let $u_{0}$ be any element of $X$, let $0 \leqq a<b \leqq T$, and let $f(t) \in L^{2}([a, b], X)$.

(i) Suppose $c(a)>0$. A weak solution of $(1.1)$ in $[a, b]$ with initial condition $u_{0}$ at $t=a$ (or with $\left.u(a)=u_{0}\right)$ is a function $u(t) \in L^{2}([a, b], X)$ such that

$$
-\left(u_{0}, c(a) \varphi(a)\right)+\int_{a}^{b}\left(u,-(c \varphi)^{\prime}+A^{*}(t) \varphi\right) d t=\int_{a}^{b}(f, \varphi) d t
$$

for every test function $\varphi(t)$ in $[a, b]$.

(ii) Suppose $c(a)=0$. A function $u(t) \in L^{2}([a, b], X)$ is said to be a weak solution of $(1.1)$ in $[a, b]$ if

$$
\int_{a}^{b}\left(u,-(c \varphi)^{\prime}+A^{*}(t) \varphi\right) d t=\int_{a}^{b}(f, \varphi) d t
$$

for every test function $\varphi$ in $[a, b]$.

For brevity, when we refer to a weak solution in $[a, b]$, we mean a solution given by either one of the Definitions 1.6(i) or 1.6(ii).

An $X$-valued function $f(t)$ defined in an interval $I$ is said to be uniformly Hölder continuous on $I$ if $|f(t)-f(s)| \leqq C|t-s|^{\beta}(0<\beta \leqq 1)$ for all $t, s$ in $I ; C, \beta$ are constants.

In the sequel, we shall denote by the same symbol $C$ any one of various different constants.

THEOREM 1.1. Let $c(t) \in \mathscr{C}_{0}, A(t) \in \mathfrak{A}_{0}$ and let $\left(\mathrm{AC}_{0}\right)$ hold. Then any weak solution of $(1.1)$ in $[0, T]$ is a classical solution in any subinterval $(a, b)$ of $(0, T)$ where $c(t)>0$ for all $a<t<b$ and $f(t)$ is uniformly Hölder continuous in $[a, b]$.

For the proof we shall need several lemmas.

LEMMA 1.1. If $u(t)$ is a weak solution in $[0, T]$, then it is also a weak solution in $\left[0, T^{\prime}\right]$ for any $0<T^{\prime} \leqq T$.

Proof. Let $\varphi(t)$ be a test function on $\left[0, T^{\prime}\right]$, and let $\zeta_{\varepsilon}(t) \in C^{\infty}[0, T]$ be such that

$$
\begin{aligned}
\zeta_{\varepsilon}(t)=1 & \text { if } 0 \leqq t<T^{\prime}-\varepsilon, \\
=0 & \text { if } t>T^{\prime}-\varepsilon / 2,
\end{aligned}
$$

$0 \leqq \zeta_{\varepsilon}(t) \leqq 1$ otherwise in $[0, T]$, and $\left|\zeta_{\varepsilon}^{\prime}(t)\right| \leqq C / \varepsilon$.

Define

$$
\begin{aligned}
\varphi_{\varepsilon}(t) & =\zeta_{\varepsilon}(t) \varphi(t) & & \text { if } 0 \leqq t \leqq T^{\prime} \\
& =0 & & \text { if } t>T^{\prime}
\end{aligned}
$$


Since $u(t)$ is a weak solution in $[0, T]$ and since $\varphi_{\varepsilon}(t)$ is a test function in $[0, T]$, we can apply (1.2) (we assume, for definiteness, that $c(0)>0$ ), and get

$$
-\left(u_{0}, c(0) \varphi(0)\right)+\int_{0}^{T^{\prime}}\left(u,-(c \varphi)^{\prime}+A^{*}(t) \varphi\right) d t=\int_{0}^{T^{\prime}}(f, \varphi) d t+I_{\delta}
$$

where

$$
\begin{aligned}
I_{\varepsilon}= & \int_{T^{\prime}-\varepsilon}^{T^{\prime}}\left(u,\left(\zeta_{\varepsilon}-1\right)(c \varphi)^{\prime}\right) d t+\int_{T^{\prime}-\varepsilon}^{T^{\prime}}\left(\zeta_{\varepsilon}^{\prime} u, c \varphi\right) d t+\int_{T^{\prime}-\varepsilon}^{T^{\prime}}\left(u,\left(1-\zeta_{\varepsilon}\right) A^{*}(t) \varphi\right) d t \\
& +\int_{T^{\prime}-\varepsilon}^{T^{\prime}}\left(f,\left(\zeta_{\varepsilon}-1\right) \varphi\right) d t \\
= & I_{\varepsilon}^{1}+I_{\varepsilon}^{2}+I_{\varepsilon}^{3}+I_{\varepsilon}^{4} .
\end{aligned}
$$

It can easily be seen that $\left|I_{\varepsilon}^{1}\right|+\left|I_{\varepsilon}^{3}\right|+\left|I_{\varepsilon}^{4}\right| \rightarrow 0$ if $\varepsilon \rightarrow 0$.

To estimate $I_{\varepsilon}^{2}$ we note that, since $\varphi\left(T^{\prime}\right)=0$ and $\varphi \in C^{1}\left(\left[0, T^{\prime}\right], X\right)$, we have $|\varphi(t)| \leqq C\left|T^{\prime}-t\right|$ for $T^{\prime}-\varepsilon \leqq t \leqq T^{\prime}$, where $C$ is independent of $\varepsilon$. Thus

$$
\left|I_{\varepsilon}^{2}\right|^{2} \leqq \frac{C}{\varepsilon^{2}} \int_{T^{\prime}-\varepsilon}^{T^{\prime}}|u|^{2} d t \int_{T^{\prime}-\varepsilon}^{T^{\prime}}\left(T^{\prime}-t\right)^{2} d t \leqq C \varepsilon .
$$

Taking, in (1.3), $\varepsilon \rightarrow 0,(1.2)$ follows with $[a, b]=\left[0, T^{\prime}\right]$.

LEMMA 1.2. If $u(t)$ is a weak solution of $(1.1)$ in $[0, T]$ and $c(a)=0$ for some $0<a<T$, then $u(t)$ is a weak solution in $[a, b]$ for any $a<b \leqq T$.

Proof. Let $\zeta_{\varepsilon}(t)$ be a $C^{\infty}$-function similar to the one constructed above, with $\zeta_{\varepsilon}(t)=0$ for $0 \leqq t \leqq a+\varepsilon / 2, \zeta_{\varepsilon}(t)=1$ for $t>a+\varepsilon$. Now apply (1.2) to $\varphi_{\varepsilon}=\zeta_{\varepsilon} \varphi$ and use arguments similar to those used in the proof of Lemma 1.1, together with the inequality $c(t) \leqq C|t-a|$. It follows that $u(t)$ is a weak solution in $[a, T]$. From the proof of Lemma 1.1 it then also follows that $u(t)$ is a weak solution in $[a, b]$.

LEMMA 1.3. If $0 \leqq a<b \leqq T, c(a)=c(b)=0, c(t)>0$ for all $a<t<b$, then there is at most one weak solution of $(1.1)$ in $[a, b]$.

Proof. Let $u(t)$ be a weak solution in $[a, b]$ with $f=0$. We have to show that $u=0$ in $[a, b]$. Let $a<\beta<b$ and let $\varphi$ be the (classical) solution of

$$
-(c \varphi)^{\prime}+A^{*}(t) \varphi=h(t) \text { in }(a, \beta], \quad \varphi(\beta)=0,
$$

for some uniformly Hölder continuous function $h(t)$. (Its existence follows by applying [4] to the equation for $\hat{\varphi}$, where $\varphi=\hat{\varphi} \exp \left[\int^{t}\left(c^{\prime} / c\right) d t\right]$.) Note that $\varphi(t)$ is not necessarily a test function in $[a, \beta]$, since it need not be continuously differentiable at $t=a$. It is, however, in $L^{2}([a, \beta], X)$. Indeed, multiplying both sides of (1.4) scalarly by $\varphi$ and integrating over $[a+\varepsilon, \beta]$ we get

$$
\frac{1}{2} c(a+\varepsilon)|\varphi(a+\varepsilon)|^{2}+\int_{a+\varepsilon}^{\beta}\left[\left(A^{*}(t) \varphi, \varphi\right)-\frac{1}{2} c^{\prime}|\varphi|^{2}\right] d t=\int_{a+\varepsilon}^{\beta}(h, \varphi) d t .
$$


Hence, using $\left(\mathrm{AC}_{0}\right)$ we get, for any $\delta>0$,

$$
\int_{a+\varepsilon}^{\beta}\left(\gamma-\frac{1}{2} c^{\prime}-\delta\right)|\varphi|^{2} d t \leqq(1 / 4 \delta) \int_{a+\varepsilon}^{\beta}|h|^{2} d t
$$

It follows that

$$
\int_{a}^{\beta}|\varphi|^{2} d t \leqq C \int_{a}^{\beta}|h|^{2} d t .
$$

We take now a function $\zeta_{\varepsilon}(t) \in C^{\infty}([a, \beta])$ such that $\zeta_{\varepsilon}(t)=0$ if $a \leqq t \leqq a+\varepsilon / 2$, $\zeta_{\varepsilon}(t)=1$ if $a+\varepsilon \leqq t \leqq \beta, 0 \leqq \zeta_{\varepsilon}(t) \leqq 1$ otherwise, and $\left|\zeta_{\varepsilon}^{t}(t)\right| \leqq C / \varepsilon$.

Using the fact (which follows from Lemma 1.2) that $u(t)$ is a weak solution in $[a, \beta]$ and noting that $\varphi_{\varepsilon}=\zeta_{\varepsilon} \varphi$ is a test function in $[a, \beta]$, we get

$$
\int_{a}^{\beta}\left(u,-\left(c \varphi_{\varepsilon}\right)^{\prime}+A^{*}(t) \varphi_{\varepsilon}\right) d t=0 .
$$

Recalling that $\varphi(t)$ solves (1.4), we deduce that

$$
\int_{a}^{\beta}\left(u, \zeta_{\varepsilon} h\right) d t+\int_{a}^{\beta} \zeta_{\varepsilon}^{\prime}(u, c \varphi) d t=0 .
$$

Since $c(t) \leqq C|t-a|,\left|\zeta_{\varepsilon}^{\prime}(t) c(t)\right| \leqq C$. Hence

$$
\begin{aligned}
\left|\int_{a}^{\beta} \zeta_{\varepsilon}^{\prime}(u, c \varphi) d t\right|^{2} & =\left|\int_{a}^{a+\varepsilon} \zeta_{\varepsilon}^{\prime}(u, c \varphi) d t\right|^{2} \\
& \leqq C \int_{a}^{a+\varepsilon}|\varphi|^{2} d t \int_{a}^{a+\varepsilon}|u|^{2} d t \rightarrow 0 \text { if } \varepsilon \rightarrow 0
\end{aligned}
$$

Noting also that

$$
\int_{a}^{\beta}\left(u, \zeta_{\varepsilon} h\right) d t \rightarrow \int_{a}^{\beta}(u, h) d t \quad \text { if } \varepsilon \rightarrow 0,
$$

we see that $u(t)$ is orthogonal in $L^{2}([a, \beta], X)$ to any uniformly Hölder continuous function $h(t)$. Consequently $u \equiv 0$ a.e. in $[a, \beta]$, for any $a<\beta<b$. It follows that $u \equiv 0$ a.e. in $[a, b]$.

Proof of Theorem 1.1. Assume first that $c(a)=c(b)=0$ and $c(t)>0$ for all $a<t<b$. For any $\varepsilon>0$ let $u_{\varepsilon}(t)$ be the (classical) solution of $c u_{\varepsilon}^{\prime}+A(t) u_{\varepsilon}=f$ in $(a+\varepsilon, b), u_{\varepsilon}(a+\varepsilon)=0$. We have, as in the proof of Lemma 1.3,

$$
\int_{a+\varepsilon}^{b}\left|u_{\varepsilon}\right|^{2} d t \leqq C
$$

where $C$ is a constant independent of $\varepsilon$. Define $\tilde{u}_{\varepsilon}(t)=u_{\varepsilon}(t)$ if $t>a+\varepsilon, \tilde{u}_{\varepsilon}(t)=0$ if $a \leqq t \leqq a+\varepsilon$.

Because of (1.5), we can extract a sequence $\left\{\tilde{u}_{\varepsilon_{m}}\right\}$ such that $\tilde{u}_{\varepsilon_{m}} \rightarrow v$ (weak convergence) in $L^{2}([a, b], X)$, and $\int_{a}^{b}|v|^{2} d t \leqq C$. Let $a<\tau_{0}<\tau_{0}+\lambda<b$. Denote by $U(t, \tau)$ the fundamental solution of $(1.1)$ in $(a, b)$ (see [4]). We then have, for any $\tau_{0}<\tau<\tau_{0}+\lambda, \tau_{0}+\lambda<t<b$,

$$
u_{\varepsilon_{m}}(t)=U(t, \tau) u_{\varepsilon_{m}}(\tau)+\int_{\tau}^{t} U(t, \sigma) f(\sigma) d \sigma
$$


provided $m$ is sufficiently large, so that $a+\varepsilon_{m}<\tau_{0}$. Integrating with respect to $\tau$ over $\left[\tau_{0}, \tau_{0}+\lambda\right]$, we get

$$
\lambda u_{\varepsilon_{m}}(t)=\int_{\tau_{0}}^{\tau_{0}+\lambda} U(t, \tau) u_{\varepsilon_{m}}(\tau) d \tau+\int_{\tau_{0}}^{\tau_{0}+\lambda} \int_{\tau}^{t} U(t, \sigma) f(\sigma) d \sigma d \tau .
$$

It can easily be seen that the right-hand side of (1.6) converges weakly in $X$. Since $\tilde{u}_{\varepsilon_{m}} \rightarrow v$ in $L^{2}([a, b], X)$, a standard argument shows that, a.e.,

$$
\lambda v(t)=\int_{\tau_{0}}^{\tau_{0}+\lambda} U(t, \tau) v(\tau) d \tau+\int_{\tau_{0}}^{\tau_{0}+\lambda} \int_{\tau}^{t} U(t, \sigma) f(\sigma) d \sigma d \tau .
$$

Dividing by $\lambda$ and taking the limit as $\lambda \rightarrow 0$ (assuming $\tau_{0}$ to be a Lebesgue point of $v(t))$, we find that

$$
v(t)=U\left(t, \tau_{0}\right) v\left(\tau_{0}\right)+\int_{\tau_{0}}^{t} U(t, \sigma) f(\sigma) d \sigma \quad \text { a.e. }
$$

It follows (by [4]) that $v(t)$ is a classical solution of $(1.1)$ in $(a, b)$.

If we show that $v(t)$ is a weak solution in $(a, b)$ then, by Lemma $1.3, v(t)=u(t)$, so that $u(t)$ is a classical solution of $(1.1)$ in $(a, b)$. We have

$$
\int_{a+\varepsilon}^{b-\varepsilon^{\prime}}\left(c v^{\prime}, \varphi\right) d t+\int_{a+\varepsilon}^{b-\varepsilon^{\prime}}(A(t) v, \varphi) d t=\int_{a+\varepsilon}^{b-\varepsilon^{\prime}}(f, \varphi) d t,
$$

for any test function $\varphi(t)$ in $[a, b]$. Hence

$$
\begin{aligned}
& c\left(b-\varepsilon^{\prime}\right)\left(v\left(b-\varepsilon^{\prime}\right), \varphi\left(b-\varepsilon^{\prime}\right)\right)-c(a+\varepsilon)(v(a+\varepsilon), \varphi(a+\varepsilon)) \\
& \quad+\int_{a+\varepsilon}^{b-\varepsilon^{\prime}}\left(v,-(c \varphi)^{\prime}+A^{*}(t) \varphi\right) d t=\int_{a+\varepsilon}^{b-\varepsilon^{\prime}}(f, \varphi) d t .
\end{aligned}
$$

Since $v(t) \in L^{2}([a, b], X)$, there is a sequence $\varepsilon_{n} \searrow 0$ such that $\left|v\left(a+\varepsilon_{n}\right)\right| \leqq 1 / \varepsilon_{n}^{2 / 3}$, say. For $c(t)$ in $[a, a+\varepsilon]$ we have the bound $|c(t)| \leqq C \varepsilon$. Consequently,

$$
\left|c\left(a+\varepsilon_{n}\right)\left(v\left(a+\varepsilon_{n}\right), \varphi\left(a+\varepsilon_{n}\right)\right)\right| \leqq C \varepsilon_{n}^{1 / 3} \rightarrow 0 \text { if } n \rightarrow \infty .
$$

Similarly

$$
\left|c\left(b-\varepsilon_{n}^{\prime}\right)\left(v\left(b-\varepsilon_{n}^{\prime}\right), \varphi\left(b-\varepsilon_{n}^{\prime}\right)\right)\right| \leqq C\left(\varepsilon_{n}^{\prime}\right)^{1 / 3} \rightarrow 0 \quad \text { if } n \rightarrow \infty,
$$

for some sequence $\varepsilon_{n}^{\prime} \searrow 0$. Here we need not even use the fact that $c(b)=0$, since $\varphi(b)=0$.

Taking $\varepsilon=\varepsilon_{n} \searrow 0, \varepsilon^{\prime}=\varepsilon_{n}^{\prime} \searrow 0$ in (1.7), we find that $v(t)$ satisfies (1.2'). We have proved Theorem 1.1 in case $c(a)=c(b)=0$.

If $c(a)>0$ and if there is a point $a^{\prime}$ such that $0 \leqq a^{\prime}<a, c\left(a^{\prime}\right)=0, c(t)>0$ in $\left(a^{\prime}, b\right)$, then again we conclude that $u(t)$ coincides in $\left(a^{\prime}, b\right)$ with the classical solution $v(t)$. Suppose next that no such $a^{\prime}$ exists. Then $c(t)>0$ in $[0, b)$. We define $v$ by

$$
c v^{\prime}+A v=f \text { in }(0, b), \quad v(0)=u_{0} .
$$


Since $v$ is continuous in $[0, b)$,

$$
c(\varepsilon)(v(\varepsilon), \varphi(\varepsilon)) \rightarrow c(0)(v(0), \varphi(0)) .
$$

It follows, by slightly modifying the previous arguments, that $v(t)$ satisfies (1.2). Hence, the weak solution $u$ coincides with $v$ in $(0, b) ; u$ is thus a classical solution in $(a, b)$.

Finally, the above proof remains unchanged in case $c(b)>0$ (see the sentence following (1.8)).

THEOREM 1.2. Suppose $c(t) \in \mathscr{C}_{0}, A(t) \in \mathfrak{A}_{0}$, and assume that the condition $\left(\mathrm{AC}_{0}\right)$ holds. Let $[a, b]$ be any subinterval of $[0, T]$ and let $u(t)$ be a weak solution of $(1.1)$ in $[0, T]$, with initial condition $u(0)=u_{0}$ in case $c(0)>0$. Then $u(t)$ is a weak solution of $(1.1)$ in $[a, b]$, with initial condition $u(a)$ at $t=a$ in case $c(a)>0$.

Proof. In view of Lemmas $1.1,1.2$ it suffices to show that $u(t)$ is a weak solution in $[a, T]$ if $a>0$ and $c(a)>0$.

Suppose first that there is a point $0 \leqq \alpha<a$ such that $c(\alpha)=0$, and $c(t)>0$ for all $\alpha<t \leqq a$. Since $u(t) \in L^{2}([\alpha, a], X)$ there is a sequence of points $t_{n} \searrow \alpha$ such that

$$
\left|u\left(t_{n}\right)\right| \leqq 1 /\left(t_{n}-\alpha\right)^{2 / 3} \text {. }
$$

Let $\varepsilon_{n}=t_{n}-\alpha$. We have the following equations for any test function $\varphi(t)$ in $[\alpha, T]$ :

$$
\int_{\alpha}^{T}\left(u,-(c \varphi)^{\prime}+A^{*}(t) \varphi\right) d t=\int_{\alpha}^{T}(f, \varphi) d t \quad \text { by Lemma 1.2, }
$$

and

$$
\int_{\alpha+\varepsilon_{n}}^{a}\left(c u^{\prime}+A(t) u, \varphi\right) d t=\int_{\alpha+\varepsilon_{n}}^{a}(f, \varphi) d t
$$

as $u(t)$ is a classical solution in $\left(\alpha, a+\varepsilon^{\prime}\right)$ for some $\varepsilon^{\prime}>0$. Integrating by parts in the last equation and then subtracting the two equations, we get

where

$$
\int_{a}^{T}\left(u,-(c \varphi)^{\prime}+A^{*}(t) \varphi\right) d t=\int_{a}^{T}(f, \varphi) d t+I_{n}
$$

$$
\begin{aligned}
I_{n}= & \int_{\alpha}^{\alpha+\varepsilon_{n}}\left(u,\left(c \varphi^{\prime}\right)-A^{*}(t) \varphi\right) d t+\int_{\alpha}^{\alpha+\varepsilon_{n}}(f, \varphi) d t+(c(a) u(a), \varphi(a)) \\
& -c\left(\alpha+\varepsilon_{n}\right)\left(u\left(\alpha+\varepsilon_{n}\right), \varphi\left(\alpha+\varepsilon_{n}\right)\right) .
\end{aligned}
$$

It is easy to see that the last two integrals tend to zero as $n \rightarrow \infty$. Next, since $c(\alpha)=0, c(t)<C \varepsilon_{n}$ if $|t-\alpha| \leqq \varepsilon_{n}$. It follows, using (1.9), that

$$
\left|c\left(\alpha+\varepsilon_{n}\right) u\left(\alpha+\varepsilon_{n}\right)\right| \leqq C \cdot \varepsilon_{n}^{1 / 3} \rightarrow 0 \text { if } n \rightarrow \infty .
$$

We conclude that $I_{n} \rightarrow c(a)(u(a), \varphi(a))$. Consequently,

$$
-(c(a) u(a), \varphi(a))+\int_{a}^{T}\left(u,-(c \varphi)^{\prime}+A^{*}(t) \varphi\right) d t=\int_{a}^{T}(f, \varphi) d t,
$$

so that $u(t)$ is a weak solution of $(1.1)$ in $[a, T]$ with initial condition $u(a)$ at $t=a$. 
If $\alpha$, as defined at the outset of the proof, does not exist, then $c(t)>0$ for $0 \leqq t \leqq a$. In that case, the above proof can, in fact, be somewhat simplified.

\section{Existence of weak solutions.}

THEOREM 2.1. If $c(t) \in \mathscr{C}_{0}, \quad A(t) \in \mathfrak{A}_{0}$ and $\left(\mathrm{AC}_{0}\right)$ holds, then, for any $f(t) \in L^{2}([0, T], X), u_{0} \in X$, there exists a weak solution to (1.1), with initial condition $u_{0}$ in case $c(0)>0$.

Proof. Put $c_{\varepsilon}(t)=c(t)+\varepsilon$ and let $u_{\varepsilon}(t)$ be the (classical) solution of

$$
c_{\varepsilon}(t) u_{\varepsilon}^{\prime}(t)+A(t) u_{\varepsilon}(t)=f(t) \quad \text { in }(0, T], \quad u_{\varepsilon}(0)=u_{0} .
$$

An argument similar to the one used in the proof of Lemma 1.3 shows that

$$
\int_{0}^{T}\left|u_{\varepsilon}\right|^{2} d t \leqq C\left\{\int_{0}^{T}|f|^{2} d t+\left|u_{0}\right|^{2}\right\}
$$

It follows that there exists a weakly convergent sequence $\left\{u_{\varepsilon_{n}}\right\}$ in $L^{2}([0, T], X)$ with some weak limit $u$.

To show that $u$ is a weak solution, we multiply both sides of (2.1) scalarly by any test function $\varphi$ in $[0, T]$ and integrate by parts. Passing to the limit, as $\varepsilon_{n} \searrow 0$, we get (1.2) (or $\left.\left(1.2^{\prime}\right)\right)$.

\section{Uniqueness.}

THEOREM 3.1. Under the assumptions of Theorem 2.1, the weak solution is unique.

Proof. We have to show that if $u(t)$ is a weak solution of (1.1) with $f=0, u_{0}=0$ then $u=0$.

In any interval $(a, b)$ where $c(t)>0$ if $a<t<b, c(a)=c(b)=0$, the weak solution is unique (by Lemma 1.3), and 0 is clearly a weak solution. Hence $u=0$ in such an interval.

Consider next an interval $(0, \alpha)$ such that $c(t)>0$ if $0 \leqq t<\alpha$ and $c(\alpha)=0$ (such an interval may exist only if $c(0)>0)$. Then, by the proof of Lemma 1.3 , the weak solution $u$ in $[0, \alpha]$ is unique, and again we conclude that $u=0$ in $[0, \alpha)$. Similarly, if there exists an interval $(\beta, T]$ such that $c(t)>0$ if $\beta<t \leqq T$ and $c(\beta)=0$, then $u=0$ in this interval.

We have shown that $u(t)=0$ in the open subset of $(0, T)$ where $c>0$. Denote by $\Delta$ the subset of $(0, T)$ where $c=0$. We shall show that $u(t)=0$ at all the Lebesgue points $t$ of $u$ which belong to $\Delta$ and at which $c^{\prime}(t)$ is continuous. This will complete the proof of the theorem.

Let $t_{0}$ be a Lebesgue point of $u(t)$ in $(0, T)$ for which $c\left(t_{0}\right)=0$ and $c^{\prime}(t)$ is continuous at $t=t_{0}$. Then $c^{\prime}\left(t_{0}\right)=0$. We take now a function $\zeta_{\varepsilon}(t) \in C^{\infty}([0, T])$ such that $\zeta_{\varepsilon}(t)=1$ if $\left|t-t_{0}\right|<\varepsilon / 2, \zeta_{\varepsilon}(t)=0$ if $\left|t-t_{0}\right|>\varepsilon, 0 \leqq \zeta_{\varepsilon}(t) \leqq 1$ otherwise, and $\left|\zeta_{\varepsilon}^{\prime}(t)\right|<C / \varepsilon$. Let $\varphi_{8}(t)=\zeta_{\varepsilon}(t) A^{*-1}\left(t_{0}\right) u\left(t_{0}\right)$ (note that $A^{*-1}(t)$ is a bounded operator). 
Then,

Now,

$$
\begin{aligned}
0= & -\int_{t_{0}-\varepsilon}^{t_{0}+\varepsilon}\left(u, c^{\prime}(t) \varphi_{\varepsilon}(t)\right) d t-\int_{t_{0}-\varepsilon}^{t_{0}+\varepsilon}\left(u, c(t) \varphi_{\varepsilon}^{\prime}(t)\right) d t+\int_{t_{0}-\varepsilon}^{t_{0}+\varepsilon}\left(u, \zeta_{\varepsilon} u\left(t_{0}\right)\right) d t \\
& +\int_{t_{0}-\varepsilon}^{t_{0}+\varepsilon}\left(u, \zeta_{\varepsilon}(f)\left[A^{*}(t)-A^{*}\left(t_{0}\right)\right] A^{*-1}\left(t_{0}\right) u\left(t_{0}\right)\right) d t \\
\equiv & -I_{1}-I_{2}+I_{3}+I_{4} .
\end{aligned}
$$

$$
\varepsilon^{-1}\left|I_{1}\right| \leqq C \sup _{\left|t-t_{0}\right|<\varepsilon}\left|c^{\prime}(t) \varphi_{\varepsilon}(t)\right| \varepsilon^{-1} \int_{t_{0}-\varepsilon}^{t_{0}+\varepsilon}|u| d t \rightarrow 0 \quad \text { if } \varepsilon \rightarrow 0,
$$

since $\left|\varphi_{\varepsilon}(t)\right| \leqq C, c^{\prime}(t) \rightarrow c^{\prime}\left(t_{0}\right)=0$ as $t \rightarrow t_{0}$. Next

$$
\varepsilon^{-1}\left|I_{2}\right| \leqq C \sup _{\left|t-t_{0}\right|<\varepsilon}\left|c(t) \varphi_{\varepsilon}^{\prime}(t)\right| \varepsilon^{-1} \int_{t_{0}-\varepsilon}^{t_{0}+\varepsilon}|u| d t \rightarrow 0 \quad \text { if } \varepsilon \rightarrow 0
$$

since $\left|\varphi_{\varepsilon}^{\prime}(t)\right| \leqq C / \varepsilon, c(t)=o\left(\left|t-t_{0}\right|\right)$.

Next, we easily deduce that $(1 / 2 \varepsilon) I_{3} \rightarrow u\left(t_{0}\right)$ if $\varepsilon \rightarrow 0$.

The second inequality in the condition (iii) defining the class $\mathfrak{A}_{0}$ implies that

$$
\left\|\left[A^{*}(t)-A^{*}(s)\right] A^{*-1}(\tau)\right\| \leqq C|t-s|^{\alpha} \quad(0<\alpha \leqq 1) .
$$

Consequently, as $\varepsilon \rightarrow 0$,

$$
\frac{1}{\varepsilon}\left|I_{4}\right| \leqq \frac{C}{\varepsilon} \int_{t_{0}-\varepsilon}^{t_{0}+\varepsilon}\left|t-t_{0}\right|^{\alpha}|u| d t \leqq C \varepsilon^{\alpha}\left[\frac{1}{2 \varepsilon} \int_{t_{0}-\varepsilon}^{t_{0}+\varepsilon}|u| d t\right] \rightarrow 0 .
$$

We conclude that $u\left(t_{0}\right)=0$ at any Lebesgue point $t_{0}$; hence $u \equiv 0$ a.e.

THEOREM 3.2. Under the assumptions of Theorem 2.1, there exists a unique solution $u$ of (1.1) and it satisfies the inequality

$$
\int_{0}^{T}|u|^{2} d t \leqq C\left\{\int_{0}^{T}|f|^{2} d t+\left|u_{0}\right|^{2}\right\}
$$

where $C$ is a constant independent of $u, u_{0}$ and $f$; if $c(0)=0$ then the term $\left|u_{0}\right|^{2}$ in (3.2) is to be dropped out.

Proof. The inequality (3.2) is a direct consequence of (2.2) and Theorem 3.1.

Remark. From Theorems 1.2, 3.1 we deduce the following: If $t_{0}$ is the first zero of $c(t)$, and if $u, \hat{u}$ are two weak solutions in $[0, T]$ with right-hand sides $f, \hat{f}$ such that $f(t)=\hat{f}(t)$ on $\left[t_{0}, T\right]$, then $u(t)=\hat{u}(t)$ on $\left[t_{0}, T\right]$.

Added in proof. Degenerate evolution equations have been considered in [8], [9]. The methods given there easily yield another proof of Theorem 3.1.

\section{Regularity.}

Definition 4.1. A function $u(t) \in L^{2}([0, T], X)$ is said to belong to the class $H^{p}([0, T], X)$ if $u(t)$ has weak derivatives in $L^{2}([0, T], X)$ up to order $p$ (see [4]).

Definition 4.2. A weak solution $u(t)$ of $(1.1)$ in $[0, T]$ is called a strong solution of $(1.1)$ in $[0, T]$ if for every $\varepsilon>0, u \in H^{1}([\varepsilon, T], X), u(t) \in D_{A}$ for all $0<t \leqq T$, $A(t) u(t) \in L^{2}([\varepsilon, T], X)$. 
Definition 4.3. An element $A(t) \in \mathfrak{A}_{0}$ belongs to the class $\mathfrak{A}_{1}$ if

(i) for any $x \in X$, the function $A(t) A^{-1}(0) x$ has a continuous derivative

$$
(d / d t)\left[A(t) A^{-1}(0) x\right] \equiv A^{\prime}(t) A^{-1}(0) x \quad \text { for all } t \in[0, T]
$$

(ii) the operator $A^{\prime}(t) A^{-1}(0)$ is uniformly bounded on $[0, T]$, and

$$
\left\|\left[A^{\prime}(t) A^{-1}(0)-A^{\prime}(s) A^{-1}(0)\right]\right\| \leqq C|t-s|^{\alpha} \quad(0<\alpha \leqq 1) ;
$$

(iii) $A(t)-c(t) A^{\prime}(t) A^{-1}(t)+c^{\prime} I$ belongs to $\mathfrak{A}_{0}$.

Note that the last condition is satisfied whenever $\|R(\lambda, A(t))\| \leqq C /(1+|\lambda|)$ for all $\lambda$ with $\operatorname{Re} \lambda \leqq \mu$, where $\mu>c(t)\left\|A^{\prime}(t) A^{-1}(t)\right\|+\left|c^{\prime}(t)\right|$.

Definition 4.4. An element $A(t) \in \mathfrak{Q}_{1}$ is said to satisfy the condition $\left(\mathrm{AC}_{1}\right)$ if $\operatorname{Re}(A(t) u, u) \geqq \gamma_{1}|u|^{2}$ for all $u \in D_{A}$, where $\gamma_{1}>\left\|A^{\prime}(t) A^{-1}(t)\right\| c(t)+(3 / 2)\left|c^{\prime}(t)\right|+\eta$ for some $\eta>0$.

THEOREM 4.1. If $c(t) \in \mathscr{C}_{0}, A(t) \in \mathfrak{A}_{1},\left(\mathrm{AC}_{1}\right)$ holds, $u_{0} \in X, f \in H^{1}([0, T], X)$, and if $u(t)$ is a weak solution of $(1.1)$ in $[0, T]$, with $u(0)=u_{0}$ in case $c(0)>0$, then $u \in C([0, T], X)$ and it is a strong solution in $[0, T]$.

Proof. Assume first that $c(0)>0$.

We give the proof first in case $c(t)$ is twice continuously differentiable.

We first assume that $f(t)$ and $f^{\prime}(t)$ are uniformly Hölder continuous in [0,T].

Since $c(0)>0, c(t)>0$ in some interval $0 \leqq t \leqq \tau$. By the proof of Theorem 1.1, $u(t)$ is a classical solution in $[0, \tau]$. If we prove that $u(t)$ is a strong solution, then it would follow by Sobolev's theorem [4] that $u(t)$ is also continuous in $[\tau, T]$, and the proof of the theorem is complete. To show that $u$ is a strong solution, consider the system

$$
\begin{gathered}
c_{\varepsilon} u_{\varepsilon}^{\prime}+A(t) u_{\varepsilon}=f, \\
u_{\varepsilon}(\tau)=u(\tau),
\end{gathered}
$$

where $c_{\varepsilon}=c(t)+\varepsilon$. Since the solution $u_{\varepsilon}(t)$ of the above system is twice continuously differentiable [4], we can differentiate both sides of (4.1). We find that $u_{\varepsilon}^{\prime}$ satisfies the system

$$
\begin{gathered}
c_{\varepsilon}\left(u_{\varepsilon}^{\prime}\right)^{\prime}+A_{1}(t, \varepsilon) u_{\varepsilon}^{\prime}=f_{1} \quad \text { in }[\tau, T] \\
u_{\varepsilon}^{\prime}(\tau)=u_{\tau, \varepsilon}
\end{gathered}
$$

where

$$
\begin{aligned}
A_{1}(t, \varepsilon) & =A(t)-c_{\varepsilon}(t) A^{\prime}(t) A^{-1}(t)+c^{\prime} I, \\
A^{\prime}(t) A^{-1}(t) & =\left(A^{\prime}(t) A^{-1}(0)\right)\left(A(0) A^{-1}(t)\right), \\
f_{1} & =f^{\prime}-A^{\prime}(t) A^{-1}(t) f(t), \\
u_{\tau, \varepsilon} & =\left(1 / c_{\varepsilon}(\tau)\right)[f(\tau)-A(\tau) u(\tau)] .
\end{aligned}
$$

It is easily seen that if $\varepsilon$ is sufficiently small then $A_{1}(t, \varepsilon) \in \mathfrak{A}_{0}$ with constants $C$ 
(in the conditions (ii), (iii) of the definition of $\mathfrak{A}_{0}$ ) independent of $\varepsilon$. Further,

$$
\begin{aligned}
\operatorname{Re}\left(A_{1}(t, \varepsilon) u, u\right) & =\operatorname{Re}(A(t) u, u)-\operatorname{Re}\left(A^{\prime}(t) A^{-1}(t) u, u\right) c_{\varepsilon}+c^{\prime}|u|^{2} \\
& \geqq\left[\gamma_{1}-\left\|A^{\prime}(t) A^{-1}(t)\right\| c_{\varepsilon}+c^{\prime}\right]|u|^{2} \geqq \gamma^{\prime}|u|^{2} \geqq\left[c^{\prime} / 2+\eta\right]|u|^{2}
\end{aligned}
$$

for some $\gamma^{\prime}>0$ and $\eta>0$ sufficiently small (and independent of $\varepsilon$ ), provided $\varepsilon$ is sufficiently small. Thus, $A_{1}(t, \varepsilon)$ satisfies $\left(\mathrm{AC}_{0}\right)$ with constants $\gamma, \eta$ independent of $\varepsilon$.

From the proof of inequality (2.2) we find that

$$
\int_{\tau}^{T}\left|u_{\varepsilon}^{\prime}\right|^{2} d t \leqq C\left\{\int_{\tau}^{T}\left|f_{1}\right|^{2} d t+\left|u_{\tau, \varepsilon}\right|^{2}\right\}
$$

Since (by Theorem 3.1) $u_{\varepsilon_{n}} \rightarrow u$ in $L^{2}([\tau, T], X)$ for some sequence $\varepsilon_{n} \searrow 0$, it follows (see, for instance, [4]) that $u$ has a weak derivative $u^{\prime}$ which is the weak limit of some subsequence of $u_{\varepsilon_{n}}^{\prime}$ in $L^{2}([\tau, T], X)$. But then $u(t) \in H^{1}([\tau, T], X)$. We have assumed so far that $f$ and $f^{\prime}$ are uniformly Hölder continuous. If $f$ is only assumed to belong to $H^{1}([0, T], X)$, then we approximate it in the $H^{1}([0, T], X)$ norm by smooth functions $f_{n}$ and denote by $u_{n}(t)$ the corresponding weak solutions. Applying the above result to each $u_{n}(t)$, and taking the limit as $n \rightarrow \infty$, we conclude that $u$, being the weak limit of $u_{n}$, is in $H^{1}([\tau, T], X)$.

It remains to prove that $u(t) \in D_{A}$ and $A(t) u(t) \in L^{2}([\tau, T], X)$. We shall need the following:

LEMMA 4.1. If $c\left(t_{0}\right)=0,0<t_{0} \leqq T$, then $u\left(t_{0}\right)=A^{-1}\left(t_{0}\right) f\left(t_{0}\right)$.

Proof. If $a \leqq t_{0} \leqq b$ and $c(t)=0$ for all $t \in[a, b]$, then $A^{-1}(t) f(t)$ is a weak solution in $[a, b]$ and by Theorems 1.2 and $3.1, u(t)$ coincides with $A^{-1}(t) f(t)$ in $[a, b]$. If $t_{0}$ is an isolated zero of $c(t)$ then, for some $\delta>0, c(t)>0$ for all $t_{0}-\delta \leqq t<t_{0}$, therefore, by Theorem 1.1, $u(t)$ is a classical solution in $\left[t_{0}-\delta, t_{0}\right)$.

The transformation

$$
\sigma(t)=\int_{t_{0}-\delta}^{t} \frac{d s}{c(s)}
$$

maps the interval $\left[t_{0}-\delta, t_{0}\right)$ onto $[0, \infty)$ and the equation for $u(t)$ into

$$
d \hat{u}(\sigma) / d \sigma+\hat{A}(\sigma) \hat{u}(\sigma)=\hat{f}(\sigma)
$$

where $\hat{u}(\sigma)=u(t)$, etc. Since $f \in H^{1}([0, T], X)$, it is (by Sobolev's theorem) Hölder continuous with any exponent $0<\alpha<1$.

The function $\hat{f}(\sigma)$ is uniformly Hölder continuous in $[0, \infty)$. For, if $t_{0}>t_{2}>t_{1}$ $\geqq t_{0}-\delta$, then

$$
\sigma_{2}-\sigma_{1}=\int_{t_{1}}^{t_{2}} \frac{d s}{c(s)} \geqq \frac{1}{M}\left(t_{2}-t_{1}\right)
$$

where $c(s) \leqq M$; it follows that

$$
\left|\hat{f}\left(\sigma_{2}\right)-\hat{f}\left(\sigma_{1}\right)\right|=\left|f\left(t_{2}\right)-f\left(t_{1}\right)\right| \leqq C\left|t_{2}-t_{1}\right|^{\alpha} \leqq C M^{\alpha}\left(\sigma_{2}-\sigma_{1}\right)^{\alpha} .
$$


Since also $\hat{f}(\infty)=\lim _{\sigma \rightarrow \infty} \hat{f}(\sigma)=f\left(t_{0}\right)$ exists, we can apply Theorem 13.1 in [4] and conclude that $\hat{u}(\infty)=\lim _{\sigma \rightarrow \infty} \hat{u}(\sigma)$ exists and equals $\hat{A}(\infty)^{-1} \hat{f}(\infty)$. Since $u(t)$ is continuous, we conclude that

$$
u\left(t_{0}\right)=\lim _{t \rtimes t_{0}} u(t)=\lim _{\sigma \rightarrow \infty} \hat{u}(\sigma)=\hat{A}^{-1}(\infty) \hat{f}(\infty)=A^{-1}\left(t_{0}\right) f\left(t_{0}\right) .
$$

Now, let $t_{0}$ be neither an isolated zero of $c(t)$ nor a point of an interval on which $c(t)$ vanishes. Then, by continuity of $c(t)$, there exists a sequence of intervals $\left(a_{n}, b_{n}\right)$ on which $c(t)>0$ and $c\left(a_{n}\right)=c\left(b_{n}\right)=0, a_{n} \rightarrow t_{0}, b_{n} \rightarrow t_{0}$. By the above result, $u\left(b_{n}\right)=A^{-1}\left(b_{n}\right) f\left(b_{n}\right)$. Hence, by continuity of $u(t)$ and of $A^{-1}(t) f(t)$, $u\left(t_{0}\right)=A^{-1}\left(t_{0}\right) f\left(t_{0}\right)$.

From Theorem 1.1 and Lemma 4.1 we conclude that $u(t) \in D_{A}$ for all $0<t \leqq T$.

From the equation (1.2) we then deduce that (1.1) is satisfied a.e. From this and from the fact that $u(t) \in H^{1}([\varepsilon, T], X)$ it follows that $A(t) u(t) \in L^{2}([\varepsilon, T], X)$.

We have assumed so far that $c(0)>0$ and that $c(t)$ is twice continuously differentiable. Suppose now that $c(0)>0$ but $c(t)$ is only piecewise continuously differentiable. Let $\tau_{1}$ be the first point of discontinuity of $c^{\prime}(t)$. We can construct a sequence $\left\{d_{m}(t)\right\}$ of twice continuously differentiable functions in $[0, T]$ such that, as $m \rightarrow \infty, d_{m}(t) \rightarrow c_{m}(t)$ uniformly in $[0, T]$ and $d_{m}^{\prime}(t) \rightarrow c^{\prime}(t)$ in $L^{1}((0, T))$. We may further assume that $d_{m}(t) \geqq 0$ in $[0, T], d_{m}(t)=c(t)$ if $0 \leqq t \leqq \tau_{1}$, and $\left(\mathrm{AC}_{1}\right)$ holds with $c$ replaced by $d_{m}$, with $\gamma_{1}$ independent of $m$. Instead of (4.3), (4.4) we write the analogous equations for the solution $v_{m, \varepsilon}$ corresponding to $d_{m}$. Then the arguments following (4.3), (4.4) show that

$$
\int_{\tau}^{T}\left|v_{m, \varepsilon}^{\prime}(t)\right|^{2} d t \leqq L_{\varepsilon}
$$

where $L_{\varepsilon}=C\left\{\int_{\tau}^{T}\left|f_{1}\right|^{2} d t+\left|v_{m, \varepsilon}(\tau)\right|^{2}\right\}$. We can take $\tau<\tau_{1}$ so that $v_{m, \varepsilon}(\tau)$ actually coincides with $u_{\tau, \varepsilon}$ (and is thus independent of $m$ ).

As easily seen, the weak limit $v_{\varepsilon}$ of the $v_{m, \varepsilon}$ is a weak solution. Hence, by Theorem $3.1, v_{\varepsilon}=u_{\varepsilon}$. From the last inequality it then follows that $\int_{\tau}^{T}\left|u_{\varepsilon}^{\prime}(t)\right|^{2} d t \leqq L_{\varepsilon}$. We can now proceed as before to complete the proof of the theorem.

It remains to prove the theorem in case $c(0)=0$. We extend the definition of $c(t), A(t), f(t)$ into $[-1,0)$ in such a way that the extended functions satisfy all the assumptions of Theorem 4.1 with respect to the interval $[-1, T]$, and $c(-1)>0$. Take a fixed point $w \in X$, and denote by $\hat{u}(t)$ the weak solution in $[-1, T]$ with $\hat{u}(-1)=w$. If we apply the above proof of Theorem 4.1 in $[-1, T]$ and then note, by the remark following Theorem 3.2 , that $u=\hat{u}$ in $[0, T]$, then the proof of Theorem 4.1 is complete also in case $c(0)=0$.

REMARK 4.1. If $c^{\prime}(t) \in L^{p}([0, T])(1 \leqq p)$ but is not piecewise continuous, then we can still define the concept of weak solution. However a weak solution is not continuous, in general, as the following example shows:

Let

$$
\begin{aligned}
c(t) & =\left(t_{0}-t\right)^{\beta} & & \text { if } 0 \leqq t \leqq t_{0}, 1-1 / p<\beta<1, \\
& =0 & & \text { if } t_{0}<t \leqq T .
\end{aligned}
$$


Define

$$
\begin{aligned}
\hat{u}(t) & =A^{-1}(t) f(t) \text { in }\left[t_{0}, T\right], \\
& =\text { any classical solution } u(t) \text { in }\left[0, t_{0}\right) .
\end{aligned}
$$

Using the transformation (4.5) we get (4.6) in the interval $\left(0, s_{0}\right)$, where $s_{0}=\sigma\left(t_{0}\right)$ $<\infty$. It is easily seen that $\lim _{t \wedge t_{0}} u(t)$ exists. Using this, one finds that $\hat{u}(t)$ is a weak solution in $[0, T]$. However, $\hat{u}\left(t_{0}+0\right)=A^{-1}\left(t_{0}\right) f\left(t_{0}\right)$ is not equal, in general, to $\hat{u}\left(t_{0}-0\right)=u\left(t_{0}-0\right)$.

Definition 4.6. An element $A(t) \in \mathfrak{A}_{0}$ is said to belong to the class $\mathscr{B}_{k}(k$ integer $\geqq 2$ ) if

(i) For any $x \in X$, the function $A(t) A^{-1}(0) x$ has continuous derivatives

$$
\left(d^{j} / d t^{j}\right)\left[A(t) A^{-1}(0) x\right] \equiv A^{(j)}(t) A^{-1}(0) x
$$

for all $1 \leqq j \leqq k$.

(ii) The operators $A^{(j)}(t) A^{-1}(0)$ are uniformly bounded for $0 \leqq t \leqq T$, and, for any $t, \tau$ in $[0, T]$,

$$
\left\|A^{(k)}(t) A^{-1}(0)-A^{(k)}(\tau) A^{-1}(0)\right\| \leqq C|t-\tau|^{\alpha} \quad(0<\alpha \leqq 1) .
$$

The proof of Theorem 4.1 can be extended to deduce the higher differentiability of the weak solution. In fact, for any positive integer $k$, there exist positive numbers $\gamma_{k}, \mu_{k}$ such that if $c(0)>0$ and

(a) $A(t) \in \mathscr{B}_{k}$ and $\|R(\lambda, A(t))\| \leqq C /(1+|\lambda|)\left(\operatorname{Re} \lambda \leqq \mu_{k}\right)$,

(b) $c(t) \in C^{k-1}[0, T]$ and $c^{(k)}(t)$ is piecewise continuous in [0,T],

(c) $\operatorname{Re}(A(t) u, u) \geqq \gamma_{k}|u|^{2}$,

(d) $f \in H^{k}([0, T], X)$,

then the weak solution belongs to $H^{k}([\varepsilon, T], X)$ for any $\varepsilon>0$.

Using Sobolev's theorem we can state

THEOREM 4.2. If $c(0)>0$ and the above conditions (a)-(d) hold, then for any $\varepsilon>0$ the weak solution $u(t)$ satisfies

(i) $u \in H^{k}([\varepsilon, T], X)$,

(ii) $A(t) u(t) \in H^{k-1}([\varepsilon, T], X)$, and

(iii) $u \in C^{k-1}((0, T], X), A(t) u(t) \in C^{k-2}((0, T], X)$.

If $u_{0} \in D\left(A^{k-1}\right)$, then $u \in C^{k}([0, T], X)$ (by [4]).

Consider now the case where $c(0)=0$, and assume that (a)-(d) hold. We can extend $A(t), f(t)$ into $[-1,0)$ in such a way that (a), (c), (d) continue to hold with respect to $[-1, T]$. As for $c(t)$, we shall assume that either $c^{(j)}(0)=0$ for $0 \leqq j \leqq k-1$ or that $c^{(j)}(0)=0$ for $0 \leqq j \leqq 2 h-1, c^{(2 h)}(0) \neq 0$, for some positive integer $h$. Then we can extend $c(t)$ into $[-1,0)$ in such a way that the extended function $c(t)$ belongs to $C^{k-1}[-1, T]$, and $c^{(k)}(t)$ is piecewise continuous in $[-1, T]$ and $c(-1)>0$. Denote by $\hat{u}$ a weak solution in $[-1, T]$. By the remark following Theorem 3.2, $u=\hat{u}$ in $[0, T]$. Hence, applying Theorem 4.2 to $\hat{u}$ in $[-1, T]$, we conclude that $u$ satisfies the assertions (i)-(iii) of Theorem 4.2 with $\varepsilon=0$. 
5. Another method of proving uniqueness. The proof of the uniqueness theorem given in $\$ 3$ does not extend to degenerate parabolic equations of the form

$$
c(x, t) \partial u / \partial t+P\left(x, t, D_{x}\right) u=f(x, t)
$$

(except when $c(x, t)$ is independent of $x$ ). Therefore, in this section we shall give another method for proving uniqueness of the weak solution of (1.1). This method will apply to the degenerate parabolic equation (5.1). However, it will require some additional assumptions on $A(t)$. These assumptions are satisfied for the parabolic case.

We shall assume

$\left(\mathrm{P}_{1}\right)$ The domain of $A^{*}(t)$ is equal to $D_{A}$ and, for every $\rho>0$, there exists a positive number $K=K(\rho)$ such that the operator $B(t) \equiv A(t)-A^{*}(t)$ satisfies $|B(t) u| \leqq \rho|A(t) u|+K|u|$ for all $u \in D_{A}$.

$\left(\mathrm{P}_{2}\right)$ The operator valued function $A^{*}(t) A^{-1}(0)$ is strongly differentiable, and its strong derivative, denoted by $\left(A^{*}(t)\right)^{\prime} A^{-1}(0)$, is a uniformly bounded operator.

(AC) The condition $\left(\mathrm{AC}_{0}\right)$ holds and $\gamma>c^{\prime}(t)+\eta$.

Theorem 5.1. Assume that $A(t) \in \mathfrak{A}_{0}, c \in \mathscr{C}_{0}$, and that $\left(\mathrm{P}_{1}\right),\left(\mathrm{P}_{2}\right),(\mathrm{AC})$ hold. If $f(t) \in L^{2}([0, T], X), c(0)>0, u_{0} \in X$ and $u(t)$ is a weak solution of $(1.1)$ with $u(0)=u_{0}$, then

$$
\int_{0}^{T} c(t)|u|^{2} d t \leqq C\left\{\left|u_{0}\right|^{2}+\int_{0}^{T}|f|^{2} d t\right\},
$$

where $C$ is a constant independent of $u, u_{0}$, and $f$.

Proof. Let $\varphi_{\varepsilon}(t)$ be the solution of

$$
-\left(c_{\varepsilon} \varphi_{\varepsilon}\right)^{\prime}+A^{*}(t) \varphi_{\varepsilon}(t)=h(t), \quad \varphi_{\varepsilon}(T)=0,
$$

for $c_{\varepsilon}=c(t)+\varepsilon$ and a given uniformly Hölder continuous function $h(t)$. Multiplying both sides of (5.3) scalarly by $\left[A^{*}(t) \varphi_{\varepsilon}(t)\right] / c_{\varepsilon}(t)$, we get

$$
\begin{gathered}
-\int_{0}^{T}\left(\varphi_{\varepsilon}^{\prime}, A^{*}(t) \varphi_{\varepsilon}\right) d t-\int_{0}^{T} \frac{c^{\prime}(t)}{c_{\varepsilon}(t)}\left(\varphi_{\varepsilon}, A^{*}(t) \varphi_{\varepsilon}\right) d t+\int_{0}^{T} \frac{\left|A^{*}(t) \varphi_{\varepsilon}\right|^{2}}{c_{\varepsilon}(t)} d t \\
=\int_{0}^{T} \frac{\left(h, A^{*}(t) \varphi_{\varepsilon}\right)}{c_{\varepsilon}(t)} d t .
\end{gathered}
$$

For any test function $\varphi_{\varepsilon}$ with $A^{*}(t) \varphi_{\varepsilon}(t)$ continuously differentiable,

$$
\begin{aligned}
-\operatorname{Re} \int_{0}^{T}\left(\varphi_{\varepsilon}^{\prime}, A^{*}(t) \varphi_{\varepsilon}\right) d t= & -\left.\frac{1}{2} \operatorname{Re}\left(A^{*}(t) \varphi_{\varepsilon}, \varphi_{\varepsilon}\right)\right|_{0} ^{T} \\
& +\frac{1}{2} \operatorname{Re} \int_{0}^{T}\left(\varphi_{\varepsilon}, A^{*^{\prime}}(t) A^{-1}(0) A(0) \varphi_{\varepsilon}\right) d t \\
& +\frac{1}{2} \operatorname{Re} \int_{0}^{T}\left(\varphi_{\varepsilon}^{\prime}, B(t) \varphi_{\varepsilon}\right) d t .
\end{aligned}
$$

By approximation, this relation holds also for any test function $\varphi_{\varepsilon}$. 
We thus get from (5.4)

$\operatorname{Re}\left(A^{*}(0) \varphi_{\varepsilon}(0), \varphi_{\varepsilon}(0)\right)+\int_{0}^{T} \operatorname{Re}\left(A^{*^{\prime}}(t) A^{-1}(0) A(0) \varphi_{\varepsilon}, \varphi_{\varepsilon}\right) d t$

$$
\begin{aligned}
+\int_{0}^{T} \operatorname{Re}\left(B(t) \varphi_{\varepsilon},\right. & \left.\varphi_{\varepsilon}^{\prime}\right) d t-2 \int_{0}^{T} \frac{c^{\prime}}{c_{\varepsilon}} \operatorname{Re}\left(A^{*}(t) \varphi_{\varepsilon}, \varphi_{\varepsilon}\right) d t+2 \int_{0}^{T} \frac{\left|A^{*}(t) \varphi_{\varepsilon}\right|^{2}}{c_{\varepsilon}} d t \\
& =2 \operatorname{Re} \int_{0}^{T} \frac{\left(A^{*}(t) \varphi_{\varepsilon}, h\right)}{c_{\varepsilon}} d t
\end{aligned}
$$

We shall now need the following inequalities:

$$
\begin{gathered}
\left\|\varphi_{\varepsilon}\right\|^{2} \leqq C\|h\|^{2}, \\
\left\|\varphi_{\varepsilon} / \sqrt{ } c_{\varepsilon}\right\|^{2} \leqq C\left\|h / \sqrt{ } c_{\varepsilon}\right\|^{2}, \\
c_{\varepsilon}\left|\varphi_{\varepsilon}^{\prime}\right|^{2} \leqq\left(C / c_{\varepsilon}\right)\left\{\left|A^{*} \varphi_{\varepsilon}\right|^{2}+\left|c^{\prime} \varphi_{\varepsilon}\right|^{2}+|h|^{2}\right\} .
\end{gathered}
$$

Here, $\|\psi\|=\left\{\int_{0}^{T}|\psi(t)|^{2} d t\right\}^{1 / 2}$.

The first inequality is proved like (1.5). Next, multiplying (5.3) by $\varphi_{\varepsilon} / c_{\varepsilon}$ we get

$$
\left\{-\frac{1}{2}\left|\varphi_{\varepsilon}\right|^{2}\right\}^{\prime}-\frac{c^{\prime}}{c_{\varepsilon}}\left|\varphi_{\varepsilon}\right|^{2}+\frac{1}{c_{\varepsilon}} \operatorname{Re}\left(\varphi_{\varepsilon}, A^{*} \varphi_{\varepsilon}\right)=\operatorname{Re} \frac{\left(\varphi_{\varepsilon}, h\right)}{c_{\varepsilon}}
$$

Proceeding similarly to the proof of (1.5) we get (5.7); the condition (AC) is hereby being used. Finally, (5.8) is obtained directly from (5.3).

We now return to (5.5). For any $\alpha>0$,

$$
\begin{aligned}
\left|\left(A^{*^{\prime}}(t) A^{-1}(0) A(0) \varphi_{\varepsilon}(t), \varphi_{\varepsilon}(t)\right)\right| & \leqq \alpha C\left|A(t) \varphi_{\varepsilon}\right|^{2}+(1 / 4 \alpha)\left|\varphi_{\varepsilon}\right|^{2} \\
& \leqq 2 \alpha C\left|A^{*}(t) \varphi_{\varepsilon}\right|^{2}+C^{\prime}\left|\varphi_{\varepsilon}\right|^{2} \quad \text { (C' constant) }
\end{aligned}
$$

by $\left(\mathrm{P}_{2}\right)$. For any $\beta>0, \rho>0$ we use the inequality $(5.8)$ and the condition $\left(\mathrm{P}_{1}\right)$ to obtain

$$
\begin{aligned}
\left|\left(B(t) \varphi_{\varepsilon}, \varphi_{\varepsilon}^{\prime}\right)\right| & \leqq \frac{1}{4 \beta} \frac{\left|B(t) \varphi_{\varepsilon}(t)\right|^{2}}{c_{\varepsilon}(t)}+\beta c_{\varepsilon}(t)\left|\varphi_{\varepsilon}^{\prime}\right|^{2} \\
& \leqq\left(\frac{\rho}{4 \beta}+C \beta\right) \frac{\left|A^{*} \varphi_{\varepsilon}\right|^{2}}{c_{\varepsilon}}+C \beta \frac{|h|^{2}}{c_{\varepsilon}}+C \beta \frac{\left|c^{\prime} \varphi_{\varepsilon}\right|^{2}}{c_{\varepsilon}}+\frac{K}{4 \beta} \frac{\left|\varphi_{\varepsilon}\right|^{2}}{c_{\varepsilon}} .
\end{aligned}
$$

Integrating over $(0, T)$ and using (5.7) we get

$$
\left.\int_{0}^{T}\left|\left(B \varphi_{\varepsilon}, \varphi_{\varepsilon}^{\prime}\right)\right| d t \leqq\left(\frac{\rho}{4 \beta}+C \beta\right) \int_{0}^{T} \frac{\left|A^{*} \varphi_{\varepsilon}\right|^{2}}{c_{\varepsilon}} d t+K^{\prime}\left\|\frac{h}{\sqrt{ } c_{\varepsilon}}\right\|^{2} \quad \text { (K' constant }\right) .
$$

Note that the constant $C$ in (5.10) is independent of $\beta, \rho$.

We now use (5.9), (5.10) and the inequalities

$$
\left|\frac{c^{\prime}}{c_{\varepsilon}}\left(A^{*} \varphi_{\varepsilon}, \varphi_{\varepsilon}\right)\right| \leqq \beta \frac{\left|A^{*} \varphi_{\varepsilon}\right|^{2}}{c_{\varepsilon}}+C_{0} \frac{\left|\varphi_{\varepsilon}\right|^{2}}{c_{\varepsilon}}, \quad \frac{\left|\left(A^{*} \varphi_{\varepsilon}, h\right)\right|}{c_{\varepsilon}} \leqq \beta \frac{\left|A^{*} \varphi_{\varepsilon}\right|^{2}}{c_{\varepsilon}}+C_{0} \frac{|h|^{2}}{c_{\varepsilon}}
$$

(where $C_{0}$ is a constant depending on $\beta$ ) in (5.5). 
Choosing $\beta$ small and then $\rho$ sufficiently small, we get, after choosing also $\alpha$ sufficiently small and using (5.6), (5.7),

$$
\int_{0}^{T} \frac{\left|A^{*} \varphi_{\varepsilon}\right|^{2}}{c_{\varepsilon}} d t \leqq C \int_{0}^{T} \frac{|h|^{2}}{c_{\varepsilon}} d t
$$

here we have used the obvious inequality $\|v\| \leqq C\left\|v / \sqrt{ } c_{\varepsilon}\right\|$ for any $v \in L^{2}([0, T], X)$. From (5.3) we get, upon using (5.7) and (5.11),

$$
\int_{0}^{T} c_{\varepsilon}\left|\varphi_{\varepsilon}^{\prime}\right|^{2} d t \leqq C \int_{0}^{T} \frac{|h|^{2}}{c_{\varepsilon}} d t
$$

We now use (1.2) with $\varphi=\varphi_{\varepsilon}$. We find that

$$
\left|\int_{0}^{T}(u, h) d t\right| \leqq \int_{0}^{T}\left|\left(f, \varphi_{\varepsilon}\right)\right| d t+\left|\left(c(0) \varphi_{\varepsilon}(0) u_{0}\right)\right|+\int_{0}^{T}\left|\left(u,\left(c-c_{\varepsilon}\right) \varphi_{\varepsilon}^{\prime}\right)\right| d t .
$$

We have

$$
\begin{aligned}
\left|c(0) \varphi_{\varepsilon}(0)\right|^{2} & =\left[\int_{0}^{T}\left(c \varphi_{\varepsilon}\right)^{\prime} d t\right]^{2} \leqq C \int_{0}^{T} \frac{|h|^{2}}{c_{\varepsilon}} d t \\
\int_{0}^{T}\left|\left(u,\left(c-c_{\varepsilon}\right) \varphi_{\varepsilon}^{\prime}\right)\right| d t & \leqq\left[\int_{0}^{T}\left|\left(\sqrt{ } c-\sqrt{ } c_{\varepsilon}\right) u\right|^{2} d t\right]^{1 / 2} \cdot\left[\int_{0}^{T} 2 c_{\varepsilon}\left|\varphi_{\varepsilon}^{\prime}\right|^{2} d t\right]^{1 / 2} \\
& \leqq C\left[\int_{0}^{T}\left|\left(\sqrt{ } c-\sqrt{ } c_{\varepsilon}\right) u\right|^{2}\right]^{1 / 2} \cdot\left[\int_{0}^{T} \frac{|h|^{2}}{c_{\varepsilon}}\right]^{1 / 2}
\end{aligned}
$$

by (5.12). Using (5.14), (5.15) and (5.6) in (5.13), we find that

$$
\begin{aligned}
\left|\int_{0}^{T}(u, h) d t\right| \leqq & C\left(\int_{0}^{T}|f|^{2} d t\right)^{1 / 2}\left(\int_{0}^{T}|h|^{2} d t\right)^{1 / 2}+C\left|u_{0}\right|\left(\int_{0}^{T} \frac{|h|^{2}}{c_{\varepsilon}} d t\right)^{1 / 2} \\
& +C \sqrt{ } \varepsilon\left(\int_{0}^{T}|u|^{2} d t\right)^{1 / 2}\left(\int_{0}^{T} \frac{|h|^{2}}{c_{\delta}} d t\right)^{1 / 2} ;
\end{aligned}
$$

the constant $C$ is independent of $h, \varepsilon$.

In the proof of (5.16) we have assumed that $h$ is uniformly Hölder continuous in $[0, T]$. However, by approximation we conclude that (5.16) holds for any $h$ in $L^{2}((0, T), X)$. Taking in particular $h(t)=c(t) u(t)$ and then letting $\varepsilon \rightarrow 0$, we get

$$
\int_{0}^{T} c(t)|u|^{2} d t \leqq C\left\{\left(\int_{0}^{T}|f|^{2} d t\right)^{1 / 2}+\left|u_{0}\right|\right\}\left\{\int_{0}^{T} c(t)|u|^{2} d t\right\}^{1 / 2}
$$

from which (5.4) follows.

Theorem 5.1 can be used to prove the uniqueness of a weak solution by a method different from that given in $\S 3$. We shall give the details in $\$ 6$, in the context of degenerate parabolic equations.

6. Application to degenerate parabolic equations. Let $\Omega$ be a bounded domain in $R^{n}$ with $\partial \Omega \in C^{2 m}$. Set $Q_{\tau}^{T}=\Omega \times(\tau, T], Q_{0}^{T} \equiv Q^{T}$. Let

$$
P\left(x, t, D_{x}\right)=\sum_{|\alpha| \leqq 2 m} a_{\alpha}(x, t) D_{x}^{\alpha}
$$

be a uniformly strongly elliptic operator in $\mathrm{Cl}\left(Q^{T}\right)$ (see [4]). 
Consider the degenerate parabolic system

$$
\begin{gathered}
c(x, t) \partial u(x, t) / \partial t+P\left(x, t, D_{x}\right) u(x, t)=f(x, t) \text { in } Q^{T}, \\
\partial^{j} u / \partial \nu_{j}=0 \text { on } \partial \Omega \times(0, T] \quad(0 \leqq j \leqq m-1), \\
u(x, 0)=g(x) \text { for } x \in \Omega
\end{gathered}
$$

( $\nu$ is the normal to $\partial Q^{T}$ ), where $c(x, t)$ is a nonnegative function.

We assume that $D_{x}^{\beta} a_{\alpha}(x, t)$ are continuous in $(x, t)$, uniformly Hölder continuous in $t$, for all $\beta \leqq \alpha$ (i.e., $\beta_{i} \leqq \alpha_{i}$ for all $i$, where $\left.\beta=\left(\beta_{1}, \ldots, \beta_{n}\right), \alpha=\left(\alpha_{1}, \ldots, \alpha_{n}\right)\right)$. We also assume that the function $c(x, t)$ is continuous and that $\partial c(x, t) / \partial t$ is piecewise continuous. In what follows $X=L^{2}(\Omega)$.

Definition 6.1. A test function in $Q^{T}$ is a function $\varphi(x, t)$ such that $D_{x}^{\alpha} \varphi(x, t) \in C\left(\mathrm{Cl}\left(Q^{T}\right)\right)$ for $0 \leqq|\alpha| \leqq 2 m, \partial \varphi / \partial t \in C\left(\mathrm{Cl}\left(Q^{T}\right)\right), \varphi(x, T)=0$ for $x \in \Omega$, and $\partial^{j} \varphi / \partial v_{j}=0$ on $\partial \Omega \times(0, T](0 \leqq j \leqq m-1)$.

Definition 6.2. A function $u(x, t) \in L^{2}([0, T], X)$ is called a weak solution of the system (6.1) if, for any test function $\varphi(x, t)$ in $Q^{T}$,

$$
\begin{aligned}
& -\int_{\Omega_{0}} c(x, 0) g(x) \varphi(x, 0) d x \\
& +\int_{0}^{T} \int_{\Omega}\left\{u(x, t) \frac{\partial}{\partial t}[-c(x, t) \varphi(x, t)]+u(x, t) P^{*}\left(x, t, D_{x}\right) \varphi(x, t)\right\} d x d t \\
& =\int_{0}^{T} \int_{\Omega} f(x, t) \varphi(x, t) d x d t,
\end{aligned}
$$

where $\Omega_{0}$ is the subset of $\Omega$ where $c(x, 0)>0$, and where $P^{*}\left(x, t, D_{x}\right)$ is the formal adjoint of $P\left(x, t, D_{x}\right)$. If $c(x, t)$ is independent of $x$, then we can apply all the results of $\S \S 1-4$. The conditions

$$
\begin{aligned}
\operatorname{Re} \int_{\Omega} u \cdot P\left(x, t, D_{x}\right) u d x \geqq \gamma \int_{\Omega}|u|^{2} d x & \text { for all } u \in C_{0}^{\infty}(\Omega), \\
& \gamma>c_{t}+\eta \quad(\gamma, \eta \text { positive constants), } \\
\|R(\lambda, A(t))\| \leqq C /(1+|\lambda|) & \text { if } \operatorname{Re} \lambda \leqq \mu \quad \text { (for a suitable } \mu)
\end{aligned}
$$

must be assumed; all the other assumptions made in these sections are satisfied (by [4]).

Consider now the case where $c(x, t)$ may depend on $x$.

The proof of the existence theorem, Theorem 2.1, easily extends to the present case. The same is true of the proof of Theorem 5.1 (the conditions $\left(\mathrm{P}_{1}\right),\left(\mathrm{P}_{2}\right)$ follow from standard estimates on elliptic operators [4]). The conditions in (6.3) always hold for some negative $\gamma, \mu$ so that even if (6.3) does not hold we can still apply the whole theory to $P\left(x, t, D_{x}\right)+k$, for all $k$ sufficiently large.

The uniqueness of the weak solution follows by combining the results of Theorem 5.1 together with some arguments used in the proof of Theorem 3.1. In fact, if $f(x, t) \equiv 0, g(x) \equiv 0$, then from Theorem 5.1 we conclude that the weak solution 
$u(x, t)$ satisfies $u(x, t) \equiv 0$ on the set where $c(x, t)>0$. Denote by $\Delta$ the set where $c(x, t)=0$. Then at almost each point $\left(x_{0}, t_{0}\right)$ of $\Delta, \partial c\left(x_{0}, t_{0}\right) / \partial t$ exists, so that $\partial c\left(x_{0}, t_{0}\right) / \partial t=0$. Hence, from (6.2),

$$
\int_{\Delta} \int u(x, t) P^{*}\left(x, t, D_{x}\right) \varphi(x, t) d x d t=0
$$

for every test function $\varphi(x, t)$ in $Q^{T}$. Now, for all $h(x, t) \in C_{0}^{\infty}\left(Q^{T}\right)$ the function $\varphi(x, t)$, which is the solution of

$$
\begin{aligned}
P^{*}\left(x, t, D_{x}\right) \varphi(x, t) & =h(x, t) \quad \text { in } Q^{T}, \\
\partial^{j} \varphi(x, t) / \partial \nu_{j} & =0 \quad \text { on } \partial \Omega \times(0, T] \quad(0 \leqq j \leqq m-1),
\end{aligned}
$$

is a test function. Hence $\int_{\Delta} \int u(x, t) h(x, t) d x d t=0$. We conclude that $u(x, t)=0$ a.e. on $\Delta$.

Once uniqueness has been established, we can proceed to prove regularity theorems, analogous to Theorems 4.1, 4.2, assuming that

$$
c(x, 0)>0 \text { for all } x \in \bar{\Omega} .
$$

We begin by noting that the assertion

$$
u_{t} \in L^{2}\left(\left[\varepsilon_{0}, T\right], X\right) \quad\left(\text { for any } \varepsilon_{0}>0\right)
$$

can be proved in the same way as in the proof of Theorem 4.1. We then deduce that $u \in C([0, T], X)$. Now apply (6.2) to a test function $\varphi(x, t)=\psi(x) \chi(t)$ and take $\chi=\chi_{n} \rightarrow \delta\left(t_{0}\right)$ where $t_{0}$ is a Lebesgue point of the function $t \rightarrow u_{t}(\cdot, t)$ from $(0, T]$ into $X$, and $\delta\left(t_{0}\right)$ is the Dirac distribution with unit mass at $t=t_{0}$. We get

$$
\begin{aligned}
\int_{\Omega} u\left(x, t_{0}\right) P^{*}\left(x, t_{0},\right. & \left.D_{x}\right) \psi(x) d x \\
& =-\int_{\Omega} c\left(x, t_{0}\right) u_{t}\left(x, t_{0}\right) \psi(x) d x+\int_{\Omega} f\left(x, t_{0}\right) \psi(x) d x
\end{aligned}
$$

Note that $u_{t}(\cdot, t) \in L^{2}(\Omega)$ for almost all $t \in(0, T)$. (6.5) and the last remark hold also for the solution $u_{\varepsilon}$ corresponding to $c=c_{\varepsilon}$. Since $u_{\varepsilon}\left(x, t_{0}\right) \in H_{0}^{m}(\Omega) \cap H^{2 m}(\Omega)$, we can deduce (by $[4$, Theorem 18.2]) that

$$
\int_{\varepsilon_{0}}^{T}\left|u_{\varepsilon}(\cdot, t)\right|_{H^{2 m}(\Omega)}^{2} d t \leqq C\left(1+\int_{\varepsilon_{0}}^{T}\left\{\left|\frac{\partial u_{\varepsilon}}{\partial t}(\cdot, t)\right|^{2}+\left|f\left(\cdot, t_{0}\right)\right|^{2}\right\} d t\right) \leqq C
$$

where $C$ is a constant independent of $\varepsilon$. Since $u_{\varepsilon} \rightarrow u$ in $L^{2}((0, T) ; X)$ we easily conclude that $u_{\varepsilon} \rightarrow u$ in $H^{2 m}(\Omega)$ for a sequence of $\varepsilon$ 's tending to zero. Hence $u(\cdot, t) \in H^{2 m}(\Omega)$ for almost all $t \in(0, T]$. Since the map $t \rightarrow u(\cdot, t)$ from $[0, T]$ into $X$ is continuous, it easily follows that $u(\cdot, t) \in H^{2 m}(\Omega)$ for all $t \in(0, T]$.

Using the facts that $u_{\varepsilon}(\cdot, t) \in H_{0}^{m}(\Omega)$ and $u_{\varepsilon} \rightarrow u$ in $L^{2}\left((0, T) ; H^{m}(\Omega)\right)$ one can also show $u(\cdot, t) \in H_{0}^{m}(\Omega)$ for almost all $t$ (by [3, p. 325, Problem 1]).

Since the function $t \rightarrow u(\cdot, t)$ from $[0, T]$ into $X$ is continuous, we easily conclude that $u(\cdot, t) \in H_{0}^{m}(\Omega)$ for all $t \in(0, T]$. 
We can now differentiate the equation for $u_{\varepsilon}$ once more with respect to $t$ and show that, for any $\varepsilon_{0}>0$,

$$
\begin{gathered}
\partial^{2} u / \partial t^{2} \in L^{2}\left(\left[\varepsilon_{0}, T\right], X\right), \quad P\left(x, t, D_{x}\right) \partial u / \partial t \in L^{2}\left(\left[\varepsilon_{0}, T\right], X\right), \\
P^{2}\left(x, t, D_{x}\right) u \in L^{2}\left(\left[\varepsilon_{0}, T\right], X\right) .
\end{gathered}
$$

Here we assume, of course, that the coefficients $a_{\alpha}, c, f$ are sufficiently smooth, and that (6.3) holds with suitable $\gamma, \mu$ (larger than before).

Proceeding in this way step by step, and using Sobolev's theorem, we obtain the following result:

THEOREM 6.1. Denote by $A(t)$ the operator in $L^{2}(\Omega)$ associated with the strongly elliptic operator $P\left(t, x, D_{x}\right)$ and the domain $H_{0}^{m}(\Omega) \cap H^{2 m}(\Omega)$. Let $a_{\alpha}, c$, f belong to $C^{\infty}\left(\mathrm{Cl}\left(Q^{T}\right)\right)$. Then, for any positive integer $k$ there exist positive numbers $\gamma_{k}, \delta_{k}$ such that, if (6.3) holds for $\gamma=\gamma_{k}, \mu=\delta_{k}$, and (6.4) holds, then the weak solution of (6.1) belongs to $C^{k}(\bar{\Omega} \times(0, T])$.

REMARK 6.1. Using the Schauder estimates for parabolic equations [3] one can obtain regularity theorems for the unique weak solution (constructed in the proof of Theorem 2.1) in every open set where $c(x, t)>0$. Such theorems establish the smoothness of the solution under weaker assumptions than in Theorem 6.1.

REMARK 6.2. If $c(x, 0)$ vanishes at some points of $\Omega$ then we can still extend the regularity theorem under some consistency assumptions. Thus, if $u_{t}(x, t)$ is to belong to $L^{2}(\Omega)$ at $t=0$, then we must have

$$
\frac{f(x, 0)-P\left(x, 0, D_{x}\right) g(x)}{c(x, 0)} \in L^{2}(\Omega) .
$$

If we assume (6.4) then we can indeed show (by the same argument as in the proof of Theorem 3.1) that $u_{t}(x, t) \in L^{2}(\Omega)$ for any $t$, and then obtain the other assertion of Theorem 6.1. Similarly we can prove higher differentiability theorems in case $c(x, 0)$ vanishes at some points of $\Omega$.

Finally, if $c(x, 0) \equiv 0$ in $\Omega$, then we extend $c, f$ and the $a_{\alpha}$ into $\bar{\Omega} \times[-1,0)$ and then apply the regularity theorems derived above for a weak solution $\hat{u}(x, t)$ in $\Omega \times[-1, T]$ (with any initial conditions). The proof of Lemma 1.2 shows that $\hat{u}(x, t)$ is a weak solution in $[0, T]$. Hence, by uniqueness, $u(x, t)=\hat{u}(x, t)$ in $Q^{T}$. We can thus draw regularity results for $u(x, t)$ in $\mathrm{Cl}\left(Q^{T}\right)$.

7. Another type of degenerate evolution equation. Consider the degenerate system

$$
u^{\prime}+c(t) A(t) u=f(t) \quad \text { in }(0, T], \quad u(0)=u_{0},
$$

where $c(t)$ is a nonnegative function.

Definition 7.1. A function $u(t) \in L^{2}([0, T], X)$ is a weak solution of (7.1) in $[0, T]$ if for any test function $\varphi(t)$ in $[0, T]$

$$
-\left(u_{0}, \varphi(0)\right)+\int_{0}^{T}\left(u,-\varphi^{\prime}+c(t) A^{*}(t) \varphi\right) d t=\int_{0}^{T}(f, \varphi) d t .
$$


It can easily be seen that a weak solution in $[0, T]$ is also a weak solution in $\left[0, T^{\prime}\right]$ for any $0<T^{\prime} \leqq T$. We shall assume that $A(t) \in \mathfrak{A}_{0}$ and that $\left(\mathrm{AC}_{0}\right)$ is satisfied for some $\gamma>0$. Note that the transformation $u \rightarrow \exp \left[k \int_{0}^{t} c(s) d s\right] u$ replaces $A(t)$ by $A(t)+k I$; hence all the results of this section remain true if only $A+k I$, for any $k \geqq 0$, is assumed to belong to $\mathfrak{A}_{0}$, and to satisfy $\operatorname{Re}((A+k I) u, u) \geqq \gamma_{0}|u|^{2}$ for some $\gamma_{0}>0$ and for all $u \in D_{A}$.

Definition 7.2. If there is a positive constant $\gamma$ such that $\operatorname{Re}(A(t) u, u) \geqq \gamma|u|^{2}$ for all $u \in D_{A}$, then we say that the condition $\left(\mathrm{A}_{0}\right)$ holds.

THEOREM 7.1. Assume that $A(t) \in \mathfrak{A}_{0}$, that $\left(\mathrm{A}_{0}\right)$ holds and that $c(t)$ is a nonnegative Hölder continuous function in $[0, T]$. Then for any $u_{0} \in X$ and $f(t) \in L^{2}([0, T], X)$ there exists a weak solution $u(t)$ of (7.1).

Proof. Proceeding as in the proof of Theorem 2.1 we see that

$$
\frac{1}{2}\left|u_{\varepsilon}(t)\right|^{2}+\operatorname{Re} \int_{0}^{t}\left(c_{\varepsilon}(t) A(t) u_{\varepsilon}, u_{\varepsilon}\right) d t=\frac{1}{2}\left|u_{0}\right|^{2}+\operatorname{Re} \int_{0}^{t}\left(f, u_{\varepsilon}\right) d t,
$$

where $u_{\varepsilon}(t)$ is the solution of

$$
u_{\varepsilon}^{\prime}+c_{\varepsilon} A(t) u_{\varepsilon}=f, \quad u_{\varepsilon}(0)=u_{0} .
$$

Hence

$$
\left|u_{\varepsilon}(t)\right|^{2} \leqq C\left\{\int_{0}^{T}|f|^{2} d t+\left|u_{0}\right|^{2}\right\}
$$

where $C$ is a constant independent of $\varepsilon$. It follows that $u_{\varepsilon_{n}} \rightarrow u$ in $L^{2}([0, T], X)$ for some sequence $\varepsilon_{n} \searrow 0$, and that $u(t)$ is a weak solution of (7.1).

We shall need, in addition to $\left(\mathrm{P}_{1}\right),\left(\mathrm{P}_{2}\right)$ (of §5), the following condition:

$\left(\mathrm{P}_{3}\right)$ There exist positive constants $k$ and $\bar{k}$ such that $\operatorname{Re}\left(x, A^{*^{\prime}}(t) x\right)$ $\leqq k \operatorname{Re}\left(x, A^{*}(t) x\right)+\bar{k}|x|^{2}$ for any $x \in D_{A}$.

THEOREM 7.2. Assume that $A(t) \in \mathfrak{A}_{0}$, that $\left(\mathrm{A}_{0}\right),\left(\mathrm{P}_{1}\right),\left(\mathrm{P}_{2}\right),\left(\mathrm{P}_{3}\right)$ hold, and that $c(t)$ is nonnegative Hölder continuous in $[0, T]$. Then the weak solution of $(7.1)$ is unique.

Proof. Let $\varphi_{\varepsilon}(t)$ be a solution of the system

$$
-\varphi_{\varepsilon}^{\prime}+c_{\varepsilon}(t) A^{*}(t) \varphi_{\varepsilon}=h(t) \text { in }[0, T), \quad \varphi_{\varepsilon}(T)=0
$$

for some uniformly Hölder continuous function $h(t)$.

Introduce $\psi_{\varepsilon}(t)=e^{k t} \varphi_{\varepsilon}(t), h_{0}(t)=e^{k t} h(t)$. Then

$$
-\psi_{\varepsilon}^{\prime}+\left[k I+c_{\varepsilon}(t) A^{*}(t)\right] \psi_{\varepsilon}=h_{0}(t) \text { in }[0, T), \quad \psi_{\varepsilon}(T)=0 .
$$

Multiplying both sides of $\left(7.5^{\prime}\right)$ scalarly by $A^{*}(t) \psi_{\varepsilon}$ and using $\left(\mathbf{P}_{1}\right),\left(\mathbf{P}_{2}\right),\left(\mathbf{P}_{3}\right)$, we find, by the method of proof of (5.11), (5.12), that

$$
\int_{0}^{T} c_{8}\left|A^{*}(t) \psi_{\varepsilon}\right|^{2} d t \leqq C \int_{0}^{T} \frac{\left|h_{0}\right|^{2}}{c_{8}} d t
$$


where $C$ is a constant independent of $h, \varepsilon$. Hence,

$$
\int_{0}^{T} c_{\varepsilon}\left|A^{*}(t) \varphi_{\varepsilon}\right|^{2} d t \leqq C \int_{0}^{T} \frac{|h|^{2}}{c_{\varepsilon}} d t
$$

From (7.5), (7.6') we get

$$
\int_{0}^{T} c_{\varepsilon}\left|\varphi_{\varepsilon}^{\prime}\right|^{2} d t \leqq C \int_{0}^{T} \frac{|h|^{2}}{c_{\varepsilon}} d t
$$

From the relation $\varphi_{\varepsilon}(0)=-\int_{0}^{T} \varphi_{\varepsilon}^{\prime}(t) d t$ and (7.5), (7.6'), we get

$$
\left|\varphi_{\varepsilon}(0)\right| \leqq C \int_{0}^{T} \frac{|h|^{2}}{c_{\varepsilon}} d t .
$$

Now let $u(t)$ be a weak solution of (7.1). Then, since $\varphi_{\varepsilon}(t)$ is a test function in $[0, T]$,

$$
\int_{0}^{T}(u, h) d t=\int_{0}^{T}\left(f, \varphi_{\varepsilon}\right) d t+\left(u_{0}, \varphi_{\varepsilon}(0)\right)+\int_{0}^{T}\left(u,\left(c-c_{\varepsilon}\right) \varphi_{\varepsilon}^{\prime}\right) d t .
$$

Using the last inequality and (7.7), we easily deduce that

$$
\begin{aligned}
\left|\int_{0}^{T}(u, h) d t\right| \leqq & C\left(\int_{0}^{T}|f|^{2} d t\right)^{1 / 2}\left(\int_{0}^{T} \frac{|h|^{2}}{c_{\varepsilon}} d t\right)^{1 / 2}+C\left|u_{0}\right|\left(\int_{0}^{T} \frac{|h|^{2}}{c_{\varepsilon}} d t\right)^{1 / 2} \\
& +C \sqrt{ } \varepsilon\left(\int_{0}^{T}|u|^{2} d t\right)^{1 / 2}\left(\int_{0}^{T} \frac{|h|^{2}}{c_{\varepsilon}} d t\right)^{1 / 2} .
\end{aligned}
$$

This inequality is the same as (5.16). We now employ the arguments following (5.16) to deduce that

$$
\int_{0}^{T} c(t)|u|^{2} d t \leqq C\left\{\int_{0}^{T}|f|^{2} d t+\left|u_{0}\right|^{2}\right\}
$$

We conclude that if $f=0, u_{0}=0$ then $u=0$ on the set where $c(t)>0$. Hence, from (7.2), $\int_{\Delta}\left(u(t), \varphi^{\prime}(t)\right) d t=0$, where $\varphi(t)$ is any test function on $[0, T]$ and $\Delta=\{t ; c(t)=0\}$. Since $u=0$ outside $\Delta$, we conclude that $\int_{0}^{T}\left(u(t), \varphi^{\prime}(t)\right) d t=0$. It easily follows that $u(t)=0$ a.e. in $[0, T]$.

Once uniqueness has been established, (7.4) gives the inequality

$$
\int_{0}^{T}|u|^{2} d t \leqq C\left\{\int_{0}^{T}|f|^{2} d t+\left|u_{0}\right|^{2}\right\}
$$

for the unique weak solution $u(t)$.

REMARK. From the uniqueness of the weak solution and from its construction (as a weak limit of the $u_{\varepsilon}$ ) we deduce, by the method of proof of Theorem 1.1, that the weak solution of (7.1) is a classical solution in every interval where $c>0$.

Similar results can be established for the degenerate parabolic case

$$
\begin{aligned}
& \partial u / \partial t+c(x, t) P\left(x, t, D_{x}\right) u=f(x, t) \text { in } Q^{T}, \\
& \partial^{j} u / \partial \nu_{j}=0 \quad \text { on } \partial \Omega \times(0, T] \quad(0 \leqq j \leqq m-1), \\
& u(x, 0)=g(x) \quad \text { in } \Omega \text {. }
\end{aligned}
$$


In this case it follows from standard theorems (for instance, by applying the Schauder estimates to $\left.u_{\varepsilon}[3]\right)$ that in every region where $c(x, t)>0, u(x, t)$ is a classical solution.

Notation.

$$
\begin{aligned}
\frac{|f|^{2}}{c} & =0 & & \text { if } c(t)=|f(t)|=0, \\
& =|f(t)|^{2} / c(t) & \text { if } c(t) & >0, \\
& =\infty & & \text { if } c(t)=0,|f(t)| \neq 0 .
\end{aligned}
$$

THEOREM 7.3. Assume that the conditions of Theorem 7.2 are satisfied and, furthermore,

(7.10) $\int_{0}^{T}\left(|f|^{2} / c\right) d t<\infty$,

(7.11) $c(t) \not \equiv 0$ in any interval $(0, \tau), \tau>0$.

Then, for any $\eta>0, u \in H^{1}([\eta, T], X)$.

Proof. We can show, by the same proof as that of (7.6), that for any $\tau \in(0, T)$,

$$
\int_{\tau}^{T} c_{\varepsilon}(t)\left|A(t) u_{\varepsilon}\right|^{2} d t \leqq C\left\{\int_{\tau}^{T} \frac{|f|^{2}}{c_{\varepsilon}} d t+\operatorname{Re}\left(u_{\varepsilon}(\tau), A(\tau) u_{\varepsilon}(\tau)\right)\right\}
$$

Suppose, now, that $c(\tau)>\delta>0$ in some interval $\tau_{0} \leqq \tau \leqq \tau_{0}+\lambda, \lambda>0$.

From (7.3) we have

$$
\int_{\tau_{0}}^{\tau_{0}+\lambda} \operatorname{Re}\left(u_{\varepsilon}(t), c_{\varepsilon}(t) A(t) u_{\varepsilon}(t)\right) d t=\frac{1}{2}\left|u_{\varepsilon}\left(\tau_{0}+\lambda\right)\right|^{2}-\frac{1}{2}\left|u_{\varepsilon}\left(\tau_{0}\right)\right|^{2}+\operatorname{Re} \int_{\tau_{0}}^{\tau_{0}+\lambda}\left(f, u_{\varepsilon}\right) d t
$$

Hence, by (7.4),

$$
\begin{aligned}
\int_{\tau_{0}}^{\tau_{0}+\lambda} \operatorname{Re}\left(u_{\varepsilon}(t), A(t) u_{\varepsilon}(t)\right) d t & \leqq \frac{1}{\delta} \int_{\tau_{0}}^{\tau_{0}+\lambda} \operatorname{Re}\left(u_{\varepsilon}, c_{\varepsilon} A u_{\varepsilon}\right) d t \\
& \leqq \frac{c}{\delta}\left\{\int_{0}^{T}|f|^{2}+\left|u_{0}\right|^{2}\right\}
\end{aligned}
$$

From inequality (7.12) we get the inequality

$$
\int_{\tau_{0}+\lambda}^{T} c_{\varepsilon}(t)\left|A(t) u_{\varepsilon}\right|^{2} d t \leqq C\left\{\int_{0}^{T} \frac{|f|^{2}}{c_{\varepsilon}} d t+\operatorname{Re}\left(u_{\varepsilon}(\tau), A(\tau) u_{\varepsilon}(\tau)\right)\right\}
$$

for any $\tau_{0} \leqq \tau \leqq \tau_{0}+\lambda$.

Integrating both sides of (7.14) with respect to $\tau$ over $\left[\tau_{0}, \tau_{0}+\lambda\right]$ and using (7.13) we get

$$
\lambda \int_{\tau_{0}+\lambda}^{T} c_{\delta}(t)\left|A(t) u_{\delta}\right|^{2} d t \leqq C\left\{\lambda \int_{0}^{T} \frac{|f|^{2}}{c_{\varepsilon}} d t+\frac{1}{\delta} \int_{0}^{T}|f|^{2} d t+\left|u_{0}\right|^{2}\right\}
$$

Since $u_{\varepsilon}^{\prime}=f-c_{\varepsilon} A(t) u_{\varepsilon}$, the last inequality implies

$$
\begin{aligned}
\lambda \int_{\tau_{0}+\lambda}^{T}\left|u_{\varepsilon}^{\prime}\right|^{2} d t & \leqq C \int_{\tau_{0}+\lambda}^{T} \frac{\left|u_{\varepsilon}^{\prime}\right|^{2}}{c_{\varepsilon}} d t \\
& \leqq C\left\{\lambda \int_{0}^{T} \frac{|f|^{2}}{c_{\varepsilon}} d t+\frac{1}{\delta} \int_{0}^{T}|f|^{2} d t+\left|u_{0}\right|^{2}\right\}
\end{aligned}
$$


We claim that, for any $\eta>0$,

$$
\int_{\eta}^{T}\left|u_{\varepsilon}^{\prime}\right|^{2} d t \leqq C\left\{\int_{0}^{T} \frac{|f|^{2}}{c_{\varepsilon}} d t+\int_{0}^{T}|f|^{2} d t+\left|u_{0}\right|^{2}\right\}
$$

where $C$ is a constant depending on $\eta$. Indeed, from (7.11) and the continuity of $c(t)$ it follows that there exists an interval $\left[\tau_{0}, \tau_{0}+\lambda\right]$ on which $c(t) \geqq \delta$ for some $\delta>0$ and $0 \leqq \tau_{0}<\tau_{0}+\lambda \leqq \eta$. But then, (7.16) follows from (7.15).

From (7.16) we conclude that $u \in H^{1}([\eta, T], X)$.

REMARK 7.1. Suppose (7.11) is not satisfied and let $\tau_{0}$ be such that $c(t) \equiv 0$ if $0 \leqq t \leqq \tau_{0}, c(t) \not \equiv 0$ in any interval $\left(\tau_{0}, \tau_{0}+\eta\right), \eta>0$. Then we can repeat the above proof with $\tau$ in some subinterval of $\left(\tau_{0}, \tau_{0}+\eta\right)$ where $c(t)>0$, and thus conclude that $u \in H^{1}\left(\left[\tau_{0}+\varepsilon, T\right], X\right)$ for any $\varepsilon>0$. Next, $u(t)=u_{0}+\int_{0}^{t} f(s) d s$ for all $0 \leqq t \leqq \tau_{0}$. Thus, $u \in H^{1}\left(\left[0, \tau_{0}\right], X\right)$. It follows that $u(t)$ is continuous in $\left[0, \tau_{0}\right) \cup\left(\tau_{0}, T\right]$, left-continuous at $\tau_{0}$. It remains to consider the right-hand continuity of $u(t)$ at $\tau_{0}$. If

$$
\left|A(\tau) u_{\varepsilon}(\tau)\right| \leqq C
$$

for some $\tau \in\left[0, \tau_{0}\right]$ and for some constant $C$ independent of $\varepsilon$, then we can still deduce from (7.12) that $u^{\prime} \in L^{2}([\tau, T], X)$. This gives the continuity of $u(t)$ at $t=\tau_{0}$.

Note that (7.17) holds, for instance, when $\tau=0$ and $u_{0} \in D_{A}$.

REMARK 7.2. Theorem 7.3 immediately applies to the system (7.9). Note that if $c(x, t)=0$ in a $\mathrm{Cl}\left(Q^{T}\right)$ neighborhood $U$ of a point $\left(x_{0}, t_{0}\right), x_{0} \in \partial \Omega$, then no boundary conditions on the weak solution $u(x, t)$ need to be given in the intersection $V$ of $U$ with the lateral boundary of $Q^{T}$. Indeed, along $V, u(x, t)=u(x, \sigma)$ $+\int_{\sigma}^{t} f(x, s) d s$ for some $\sigma$.

REMARK 7.3. Lemma 1.1 clearly extends to the degenerate evolution equation (7.1). If one a priori knows that the weak solution $u(t)$ is continuous at a point $t=a$, then one can also prove that $u(t)$ is a weak solution in $[a, T]$. Consequently, under the assumptions of Theorem 7.3, the assertion of Theorem 1.1, for the equation (7.1), remains valid.

8. General degenerate evolution equations. We consider now the general case

$$
c_{1}(t) v^{\prime}+c_{2}(t) A(t) v=g \quad \text { in }(0, T], \quad v(0)=v_{0},
$$

where $c_{1}(t) \geqq 0, c_{2}(t) \geqq 0, c_{1}+c_{2}>0$ in $[0, T]$. A weak solution of $(8.1)$ is a function $v \in L^{2}([0, T], X)$ satisfying

$$
\left(c_{1}(0) v_{0}, \varphi(0)\right)+\int_{0}^{T}\left(v,-\left(c_{1} \varphi\right)^{\prime}+c_{2}(t) A^{*}(t) \varphi\right) d t=\int_{0}^{T}(g, \varphi) d t
$$

for every test function $\varphi(t)$ in $[0, T]$. (If $c_{1}(0)=0$ then the first term on the left-hand side of (8.2) is to be dropped.) 
Without loss of generality we may assume that $c_{1}+c_{2}=1$. We can therefore rewrite (8.1) in the form

$$
c(t) v^{\prime}+(1-c(t)) A(t) v=g \quad \text { in }(0, T], \quad 0 \leqq c \leqq 1, \quad v(0)=v_{0} .
$$

We assume that $A(t) \in \mathfrak{A}_{0}$ and that (AC) holds. If we substitute $v=e^{k t} u$ into (8.3) (where $k>\gamma, \gamma$ as in (AC)), then we get

$$
c u^{\prime}+[k c I+(1-c) A(t)] u=f, \quad u(0)=u_{0}=v_{0},
$$

where $f=e^{-k t} g$.

We consider next the system

$$
c_{\varepsilon} u_{\varepsilon}^{\prime}+\left[k c_{\varepsilon} I+(1-c)_{\varepsilon} A(t)\right] u_{\varepsilon}=f, \quad u_{\varepsilon}(0)=u_{0},
$$

where $c_{\varepsilon}=c+\varepsilon,(1-c)_{\varepsilon}=1-c+\varepsilon$.

We get, as in the proof of Theorem 2.1,

$$
\int_{0}^{T}\left[k c \gamma+(1-c) \gamma-c^{\prime}-\delta\right]\left|u_{\varepsilon}\right|^{2} d t \leqq \frac{c}{4 \delta} \int_{0}^{T}|f|^{2} d t+C\left|u_{0}\right|^{2}
$$

so that

$$
\int_{0}^{T}\left|u_{\varepsilon}\right|^{2} d t \leqq C\left\{\int_{0}^{T}|f|^{2} d t+\left|u_{0}\right|^{2}\right\}
$$

From (8.6) follows the existence of a weak solution.

To prove uniqueness, we proceed as follows: Assume, first, that $c(0)<\frac{1}{2}$. We denote by $t_{1}$ the first positive point such that $c\left(t_{1}\right)=\frac{1}{2} ; t_{2}$, the first point where $c\left(t_{2}\right)=\frac{1}{4}$ and $t_{2}>t_{1} ; t_{3}$, the first point where $c\left(t_{3}\right)=\frac{1}{2}$ and $t_{3}>t_{2}$. We proceed in this way to define $t_{4}, t_{5}$, etc. After a finite number $m$ of such steps, we arrive at a point $t_{m}$ such that either $c\left(t_{m}\right)=\frac{1}{4}$ and $c(t) \leqq \frac{1}{2}$ for all $t_{m} \leqq t \leqq T$, or $c\left(t_{m}\right)=\frac{1}{2}$ and $c(t) \geqq \frac{1}{4}$ for all $t_{m} \leqq t \leqq T$. We set $t_{0}=0, t_{m+1}=T$.

We next construct a function $\tilde{c}(t)$ such that $\tilde{c}(t)=c(t)$ if $t$ is in any of the intervals $\left[t_{2 j}, t_{2 j+1}\right]$ (where $\left.c(t) \leqq \frac{1}{2}\right), \tilde{c}(t)$ is as smooth as $c(t)$ in $[0, T]$, and $\frac{1}{4} \leqq \tilde{c}(t) \leqq \frac{3}{4}$ if $t$ is in any of the intervals $\left(t_{2 j-1}, t_{2 j}\right)$.

Now let $t$ belong to an interval $\left(t_{2 j}, t_{2 j+1}\right)$. Since $1-c(t) \geqq \frac{1}{2}$, we can proceed as in $\S 5$ to derive an estimate similar to (5.11). Here $\varphi_{\varepsilon}$ is a solution of

$$
-\left(c_{\varepsilon} \varphi_{\varepsilon}\right)^{\prime}+\left[k c_{\varepsilon}+(1-c)_{\varepsilon} A^{*}(t)\right] \varphi_{\varepsilon}=h \quad \text { in }[0, T), \quad \varphi_{\varepsilon}(T)=0 .
$$

Multiplying the equation for $\varphi_{\varepsilon}$ by $A^{*} \varphi_{\varepsilon} / \tilde{c}_{\varepsilon}$ we get

$$
-\left(\left(c_{\varepsilon} \varphi_{\varepsilon}\right)^{\prime}, A^{*} \varphi_{\varepsilon} / \tilde{c}_{\varepsilon}\right)+k c_{\varepsilon}\left(\varphi_{\varepsilon}, A^{*} \varphi_{\varepsilon}\right) / \tilde{c}_{\varepsilon}+(1-c)_{\varepsilon}\left|A^{*} \varphi_{\varepsilon}\right|^{2} / \tilde{c}_{\varepsilon}=\left(h, A^{*} \varphi_{\varepsilon} / \tilde{c}_{\varepsilon}\right)
$$

here $\tilde{c}_{\varepsilon}=\tilde{c}+\varepsilon$. 
Using the second part of the identity

$$
\begin{aligned}
& \left(c_{\varepsilon} \varphi_{\varepsilon}, \frac{A^{*} \varphi_{\varepsilon}}{\tilde{c}_{\varepsilon}}\right)^{\prime} \\
& =\left(\left(c \varphi_{\varepsilon}\right)^{\prime}, \frac{A^{*} \varphi_{\varepsilon}}{\tilde{c}_{\varepsilon}}\right)+\left(\frac{c_{\varepsilon} \varphi_{\varepsilon}}{\tilde{c}_{\varepsilon}}, A^{* \prime} \varphi_{\varepsilon}\right)+\left(\frac{c_{\varepsilon}}{\tilde{c}_{\varepsilon}} \varphi_{\varepsilon}, A^{*} \varphi_{\varepsilon}^{\prime}\right)-\frac{\tilde{c}^{\prime}}{\tilde{c}_{\varepsilon}^{2}}\left(c_{\varepsilon} \varphi_{\varepsilon}, A^{*} \varphi_{\varepsilon}\right) \\
& =2 \operatorname{Re}\left(\left(c_{\varepsilon} \varphi_{\varepsilon}\right)^{\prime}, \frac{A^{*} \varphi_{\varepsilon}}{\tilde{c}_{\varepsilon}}\right)-\frac{c_{\varepsilon}}{\tilde{c}_{\varepsilon}}\left(B \varphi_{\varepsilon}, \varphi_{\varepsilon}^{\prime}\right) \\
& \quad-\frac{c^{\prime}}{\tilde{c}_{\varepsilon}}\left(A^{*} \varphi_{\varepsilon}, \varphi_{\varepsilon}\right)+\frac{c_{\varepsilon}}{\tilde{c}_{\varepsilon}}\left(\varphi_{\varepsilon}, A^{* \prime} \varphi_{\varepsilon}\right)-\frac{\tilde{c}^{\prime}}{\tilde{c}_{\varepsilon}^{2}}\left(c_{\varepsilon} \varphi_{\varepsilon}, A^{*} \varphi_{\varepsilon}\right)
\end{aligned}
$$

to express the first term of the real part on the left-hand side of (8.7). We get, upon integrating (8.7) over $\left[t_{2 j} ; t_{2 j+1}\right]$, and using $\left(\mathrm{P}_{1}\right),\left(\mathrm{P}_{2}\right),\left(\mathrm{P}_{3}\right)$,

$$
C^{\prime} \int_{t_{2 j}}^{t_{2 j+1}}(1-c)_{\varepsilon} \frac{\left|A^{*} \varphi_{\varepsilon}\right|^{2}}{\tilde{c}_{\varepsilon}} d t \leqq C^{\prime \prime} \int_{t_{2 j}}^{t_{2 j+1}} \frac{|h|^{2}}{\tilde{c}_{\varepsilon}(1-c)_{\varepsilon}} d t+\left.\left(c_{\varepsilon} \varphi_{\varepsilon}, \frac{A^{*} \varphi_{\varepsilon}}{\tilde{c}_{\varepsilon}}\right)\right|_{t_{2 j}} ^{t_{2 j+1}}
$$

where $C^{\prime}, C^{\prime \prime}$ are positive constants independent of $\varepsilon$. Note that $(1-c)_{\varepsilon}$, occurring in the integrands in (8.9), satisfies $\frac{1}{2} \leqq(1-c)_{\varepsilon} \leqq \frac{5}{6}$ if $\varepsilon$ is sufficiently small.

Next, we consider an interval $\left[t_{2 j-1}, t_{2 j}\right]$ and proceed as in the proof of Theorem 7.2. Without loss of generality we may assume that $k$ is such that $k c(t)>\tilde{c}^{\prime}(t) / \tilde{c}(t)$ in any interval $\left[t_{2 j-1}, t_{2 j}\right]$. If we multiply both sides of the equation for $\varphi_{\varepsilon}$ scalarly by $A^{*} \varphi_{\varepsilon} / \tilde{c}_{\varepsilon}$, then we obtain (8.7).

We now use the first equation of (8.8) to express the first term on the left-hand side of (8.7). The second and the fourth terms on the right-hand side of the first equation of (8.8) are easily estimated; in estimating the second term we use $\left(\mathrm{P}_{3}\right)$. (The $k$ in (8.7) is taken to be sufficiently large.)

As for the third term, we have

$$
\left(c_{\varepsilon} \varphi_{\varepsilon} / \tilde{c}_{\varepsilon}, A^{*} \varphi_{\varepsilon}^{\prime}\right)=\left(A^{*} \varphi_{\varepsilon}, c_{\varepsilon} \varphi_{\varepsilon}^{\prime}\right) / \tilde{c}_{\varepsilon}+\left(B(t) \varphi_{\varepsilon}, c_{\varepsilon} \varphi_{\varepsilon}^{\prime}\right) / \tilde{c}_{\varepsilon}
$$

The last term on the right can be estimated as before (in the proof of Theorem 7.2). As for the first term,

$$
\begin{aligned}
& \left(A^{*} \varphi_{\varepsilon} / \tilde{c}_{\varepsilon}, c_{\varepsilon} \varphi_{\varepsilon}^{\prime}\right) \\
& \quad=-\left(A^{*} \varphi_{\varepsilon}, c_{\varepsilon}^{\prime} \varphi_{\varepsilon}\right) / \tilde{c}_{\varepsilon}+\left(A^{*} \varphi_{\varepsilon},\left[k c_{\varepsilon}+(1-c)_{\varepsilon} A^{*}\right] \varphi_{\varepsilon}\right) / \tilde{c}_{\varepsilon}-\left(A^{*} \varphi_{\varepsilon}, h\right) / \tilde{c}_{\varepsilon}
\end{aligned}
$$

by the differential equation for $\varphi_{\varepsilon}$.

We take $k$ such that $k c_{\varepsilon}>c_{\varepsilon}^{\prime}$ and then obtain the lower bound $-\left(A^{*} \varphi_{\varepsilon}, h\right) / \tilde{c}_{\varepsilon}$ for the right-hand side of (8.11). Combining this with (8.10), we get a lower bound on the left-hand side of (8.10). This yields a lower bound for $\left(\left(c_{\varepsilon} \varphi_{\varepsilon}\right)^{\prime}, A^{*} \varphi_{\varepsilon} / \tilde{c}_{\varepsilon}\right)$. We can now easily deduce from (8.7) the inequality (cf. the proof of Theorem 7.2)

$$
\bar{C} \int_{t_{2 j-1}}^{t_{2 j}} \frac{(1-c)_{\varepsilon}}{\tilde{c}_{\varepsilon}}\left|A^{*} \varphi_{\varepsilon}\right|^{2} d t \leqq \overline{\bar{C}} \int_{t_{2 j-1}}^{t_{2 j}} \frac{|h|^{2}}{\tilde{c}_{\varepsilon}(1-c)_{\varepsilon}} d t+\left.\left(c_{\varepsilon} \varphi_{\varepsilon}, \frac{A^{*} \varphi_{\varepsilon}}{\tilde{c}_{\varepsilon}}\right)\right|_{t_{2 j-1}} ^{t_{2 j}} .
$$


Adding up the inequalities (8.9), (8.12), we get

$$
\int_{0}^{T} \frac{(1-c)_{\varepsilon}}{\tilde{c}_{\varepsilon}}\left|A^{*} \varphi_{\varepsilon}\right|^{2} d t \leqq C \int_{0}^{T} \frac{|h|^{2}}{\tilde{c}_{\varepsilon}(1-c)_{\varepsilon}} d t .
$$

Since $\varphi_{\varepsilon}$ is a test function,

$$
\begin{aligned}
\int_{0}^{T}(u, h) d t= & \int_{0}^{T}\left(u,\left[(1-c)_{\varepsilon}-(1-c)\right] A^{*} \varphi_{\varepsilon}-\left(c_{\varepsilon}-c\right) \varphi_{\varepsilon}^{\prime}\right) d t \\
& +\int_{0}^{T}\left(f, \varphi_{\varepsilon}\right) d t+c_{\varepsilon}(0)\left(u_{0}, \varphi_{\varepsilon}(0)\right) .
\end{aligned}
$$

The first integral on the right is bounded by

$$
C \sqrt{ } \varepsilon\left(\int_{0}^{T}|u|^{2} d t\right)^{1 / 2}\left(\int_{0}^{T} \frac{|h|^{2}}{\tilde{c}_{\varepsilon}(1-c)_{\varepsilon}} d t\right)^{1 / 2}
$$

this can be seen by breaking this integral into portions $\int_{t_{2 j}}^{t_{2 j+1}}$ and $\int_{t_{2 j-1}}^{t_{2 j}}$, and treating them as in $\$ \S 5$ and 7 respectively.

We can choose, now, $h(t)=c(t)(1-c(t)) u(t)$ and then obtain the inequality

$$
\int_{0}^{T} c(1-c)|u|^{2} d t \leqq C\left\{\int_{0}^{T}|f|^{2} d t+\left|u_{0}\right|^{2}\right\}
$$

From this inequality we can derive the uniqueness of the weak solution by the same argument as in $\$ \$ 6,7$.

In the above proof we assumed that $c(0)<\frac{1}{2}$. If $c(0) \geqq \frac{1}{2}$, the proof is similar.

We sum up:

TheOREM 8.1. Assume $A(t) \in \mathfrak{A}_{0}, c(t) \in \mathscr{C}_{0}$, and $(\mathrm{AC}),\left(\mathrm{P}_{1}\right),\left(\mathrm{P}_{2}\right),\left(\mathrm{P}_{3}\right)$ hold. Then there exists a unique weak solution $v(t)$ of $(8.1)$ for any $f(t) \in L^{2}([0, T], X)$ (and $u_{0} \in X$, if $\left.c(0)>0\right)$. Furthermore,

$$
\int_{0}^{T}|v|^{2} d t \leqq C\left\{\int_{0}^{T}|f|^{2} d t+\left|u_{0}\right|^{2}\right\}
$$

in case $c(0)=0$, the term $\left|u_{0}\right|^{2}$ in (8.14) drops out.

Theorem 8.1 can be applied to degenerate parabolic equations in a cylinder $\Omega \times(0, T]$ provided $c(x, t)$ is independent of $x$. If $c(x, t)$ depends on $x$, then we have to assume that the set of zeros of $c(x, t)$ has the form $\bigcup_{\alpha \in A}\left(\Omega \times\left\{t_{\alpha}\right\}\right)$, where $A$ is a subset of $[0, T]$.

\section{REFERENCES}

1. G. Fichera, Sulle equazioni differenziali lineari ellitico-paraboliche del secondo ordine, Atti Accad. Naz. Lincei Cl. Sci. Fis. Mat. Nat. Sez. I (8) 5 (1956), 1-30. MR 19, 658; 1432.

2. - On a unified theory of boundary value problems for elliptic-parabolic equations of second order, Boundary Problems in Differential Equations, Univ. of Wisconsin Press, Madison, Wis., 1960, pp. 97-120. MR 22 \#2789. 
3. A. Friedman, Partial differential equations of parabolic type, Prentice-Hall, Englewood Cliffs, N. J., 1964. MR 31 \#6062.

4. - Partial differential equations, Holt, Rinehart and Winston, New York, 1969.

5. V. P. Gluško and S. G. Kreĭn, Degenerating linear differential equations in Banach space, Dokl. Akad. Nauk SSSR 181 (1968), 784-787=Soviet Math. Dokl. 9 (1968), 919-922. MR 38 \#393.

6. J. J. Kohn and L. Nirenberg, Degenerate elliptic-parabolic equations of second order, Comm. Pure Appl. Math. 20 (1967), 797-872. MR 38 \#2437.

7. O. A. Oley̆nik, On linear equations of the second order with a nonnegative characteristic form, Mat. Sb. 69 (111) (1966), 111-140. (Russian) MR 33 \#1603.

8. C. Bardos and H. Brezis, Sur une classe de problèmes d'évolution non linéaires, J. Differential Equations 6 (1969), 345-394. MR 39 \#3355.

9. H. Brezis, On some degenerate nonlinear parabolic equations, Proc. Sympos. Pure Math., vol. 18, Amer. Math. Soc., Providence, R. I., 1970, pp. 28-38.

NORTHWESTERN UNIVERSITY, EvANSTON, ILLINOIS 60201 